Review

\title{
Mediterranean Diet and Cardiodiabesity: A Review
}

\author{
Elena García-Fernández ${ }^{1,2}$, Laura Rico-Cabanas ${ }^{1,2}$, Nanna Rosgaard ${ }^{1}$, Ramón Estruch ${ }^{1,3,4,5}$ \\ and Anna Bach-Faig ${ }^{1,2,6, *}$
}

1 Mediterranean Diet Foundation, Barcelona 08021, Spain;

E-Mails: elenagfdz@gmail.com (G.-F.E.); feinalaura@gmail.com (R.-C.L.); nanna2188@hotmail.com (R.N.); restruch@clinic.ub.es (E.R.)

2 Department of Health Sciences, Catalunya Open University (UOC), Barcelona 08018, Spain

3 RETIC Healthy Nutrition in Primary Prevention of Chronic Diseases: Predimed Network (RD06/0045), Barcelona 08036, Spain

4 Department of Internal Medicine, Hospital Clinic, August Pi Sunyer Biomedical Research Institute (IDIBAPS), Barcelona 08036, Spain

5 Physiopathology of Obesity and Nutrition Center (CIBEROBN), Carlos III Health Institute, Madrid 28029, Spain

6 Food and Nutrition Area, Barcelona Official College of Pharmacists, Barcelona 08009, Spain

* Author to whom correspondence should be addressed; E-Mail: abach@fdmed.org; Tel.: +34-636-023-737; Fax: +34-932-099-407.

Received: 1 May 2014; in revised form: 8 July 2014 / Accepted: 7 August 2014 /

Published: 4 September 2014

\begin{abstract}
Cardiodiabesity has been used to define and describe the well-known relationship between type 2 Diabetes Mellitus (T2DM), obesity, the metabolic syndrome (MetS) and cardiovascular disease (CVD). The objective of this study was to perform a scientific literature review with a systematic search to examine all the cardiovascular risk factors combined and their relationship with adherence to the Mediterranean Diet (MedDiet) pattern as primary prevention against cardiodiabesity in a holistic approach. Research was conducted using the PubMed database including clinical trials, cross-sectional and prospective cohort studies. Thirty-seven studies were reviewed: fourteen related to obesity, ten to CVD, nine to MetS, and four to T2DM. Indeed 33 provided strong evidence on the association between adherence to a MedDiet and a reduced incidence of collective cardiodiabesity risk in epidemiological studies. This scientific evidence makes the MedDiet pattern very useful for preventive strategies directed at the general population and also
\end{abstract}


highlights the need to consider all these diet-related risk factors and health outcomes together in daily primary care.

Keywords: Mediterranean Diet; cardiodiabesity; systematic review; primary prevention; obesity; cardiovascular disease; diabetes mellitus; metabolic syndrome

\section{Introduction}

Cardiodiabesity is a hybrid term used to define and describe the well-known relationship between type-2 diabetes mellitus (T2DM), obesity, metabolic syndrome (MetS) and cardiovascular disease (CVD). Diabesity was first coined by former US Surgeon General C. Everett Koop [1] trying to explain that there were many risk factors to T2DM besides fatness. In 2008, in the British Journal of Cardiology [2], cardiologists started to mention the idea that diabetes should be viewed as a vascular disease from the outset. The term cardiodiabesity is not only useful to describe this framework but also to highlight the need to consider all the risk factors and health outcomes combined in clinical practice.

More than 347 million people worldwide have T2DM. It is foreseen that it will be the seventh leading cause of death by 2030 [3]. As with T2DM, obesity has reached epidemic proportions globally; from 1980 to 2008 the prevalence of obesity has doubled in the world, and at least 2.8 million people die each year as a result of being overweight or obese [4]. Furthermore, CVD was the cause of three out of ten deaths, accounting for nearly 17 million deaths in 2011 [5].

Based on this global framework, the European Society of Cardiology-European Association for the Study of Diabetes (ESC-EASD) established the close relationship between hyperglycemia and microvascular disease in their guidelines. The clustering of vascular risk seen in association with insulin resistance has led to the view that cardiovascular risk appears early, prior to the development of T2DM. This fact highlights that patients with T2DM should be managed as patients with established atherosclerotic disease [6].

Moreover, the guidelines of the National Institute for Health and Clinical Excellence (NICE) recommend treatment of dyslipidemia as a key modifiable risk factor in patients with T2DM and CVD [7,8]. Furthermore, it is well known that in central obesity, visceral adiposity tissue dysfunction leads to T2DM and CVD through increased peripheral insulin resistance due to high serum insulin concentrations. This clustering of vascular risk factors (insulin resistance, visceral or abdominal obesity and dyslipidemia) among other factors (hypertension) shapes the metabolic syndrome (MetS) $[9,10]$. These metabolic disturbances have an impact not only on glucose metabolism and the cardiovascular system, but also on gout and systemic inflammation, and predispose pancreatic tissue to pre-diabetes or diabetes itself [11]. Management of obesity and prevention of CVD and T2DM should be aimed at reducing the overall risk in a holistic health prevention and management approach.

There is substantial scientific evidence that diet plays an important role in the development of numerous chronic diseases. The Mediterranean Diet (MedDiet) has long been reported to be a prudent dietary pattern for non-communicable disease prevention [12-16], linked with nutritional adequacy [17] and has been promoted as a model of healthy eating based on its relation with 
preserving a good health status and quality of life $[12,13]$, mainly through its favorable effects on cardiovascular risk factors and ultimately, by reducing cardiovascular morbidity and mortality [13].

This dietary pattern as described in the Mediterranean Diet pyramid [18] is generally characterized by a high consumption of plant foods (such as fruit, vegetables, legumes, nuts and seeds and cereals, preferably wholegrain); the seasonal choice of fresh and locally grown produce as far as possible; the presence of fruit as the main daily dessert and olive oil as the main source of dietary lipids; moderate consumption of dairy products (mainly cheese and yoghurt); low to moderate amounts of fish, poultry and eggs; consumption of red meat at a low frequency and in small amounts; and a moderate intake of wine during meals. Regarding the nutritional value of the MedDiet, this dietary pattern is low in saturated and trans fats, with an optimal nutritional quality due to the presence of healthy fats from olive oil, nuts and fish, as well as complex carbohydrates, micronutrients, antioxidants, non-nutritive factors and, furthermore, its abundant fiber and varied plant-based composition with sufficient protein intake of both plant and animal origin. The MedDiet pattern also gathers a proper ratio between the macronutrients, low energy density and low glycemic index meals $[18,19]$.

The dietary pattern analysis approach has been imposed over the single nutrient or food approach [20,21]. Many MedDiet indexes [21] have been developed to study the relationship between the MedDiet pattern and different health parameters.

Not only the MedDiet has been stated as a health model, but also a cultural model after its recognition as an Intangible Cultural Heritage of Humanity by the United Nations Educational, Scientific and Cultural Organization (UNESCO) [22]. It is a culturally accepted and highly palatable dietary pattern that allows high compliance, food availability and affordability in the Mediterranean countries. Besides, the MedDiet pattern results in lower environmental footprints than the larger extended Western diet due to the greater emphasis on plant- over animal-derived products [23]. Among the scarce literature focused on evaluating the relationship between food costs and adherence to different food patterns [24], the MedDiet pattern is flexible and adjustable according to specific needs and preferences. Thus, the MedDiet is an extremely healthy, economically affordable and environmentally sustainable food model, especially in Mediterranean countries with a higher availability of MedDiet products. However, there has been a decrease in the adherence to the MedDiet in Mediterranean countries in the last decades [25-27], occurring in parallel to the "westernization" of the society (fast food, sedentary lifestyles, etc.). Although the relationship between these dietary and lifestyle changes and the increased prevalence of different illnesses has not been well established, there is a clear parallel trend between a higher prevalence of obesity, CVD or T2DM worldwide and the aforementioned changes in the dietary patterns of the population.

Although reviews of each specific disease such as obesity, T2DM or CVD in relation to the MedDiet have been published [13,15,28-31], up to now no review of the clinical trials, cross-sectional and prospective cohort studies has been performed to examine all these risk factors combined and their relationship with the MedDiet. Therefore, the aim of this review was to introduce the term cardiodiabesity and systematically summarize scientific evidence concerning the association between adherence to the MedDiet and the collective cardiodiabesity risk in all the studies. 


\section{Materials and Methods}

The literature review was focused on prospective cohort, cross-sectional and clinical trial studies on the association between adherence to a MedDiet and cardiodiabesity, i.e., MetS, T2DM, obesity and CVD as primary prevention outcomes. We excluded case-control design studies because of their related high potential selection bias.

A systematic search was conducted up to September 2013 through a computer-assisted published data search (PubMed; MEDLINE, National Library of Medicine, Bethesda, MD, USA). In PubMed, the MeSH terms used were "Mediterranean diet" along with other key words: "Diabetes Mellitus", "Coronary Disease", "Myocardial Ischemia", "Heart Diseases", "Metabolic Syndrome X", and "Obesity". The search was limited to human studies and restricted to articles in English. All studies with full text were considered. The initial search resulted in 740 articles. The search was then narrowed to include only articles examining the effect on the four outcomes (T2DM, obesity, CVD and MetS) as the main outcome and evaluating adherence to the MedDiet pattern as a whole. Studies focused on primary prevention of outcome events were selected. Additional publications were identified from references provided in original papers. The relevance of the studies was assessed with a hierarchical approach on the basis of title, abstract and the full manuscript.

A total of 740 articles were selected with these Mesh terms and then analyzed. The topic distribution was 80 for MetS, 170 for obesity, 108 for T2DM and 382 for CVD. Among the original research articles, 523 were excluded on the basis of the title and including reviews. From the total of 217 articles and on the basis of abstracts, 122 were excluded, resulting in 95 articles: 11 on MetS, 21 on obesity, 20 on T2DM and 43 on CVD. After reading the whole article, 37 were selected, resulting in the final inclusion of 14 studies on obesity, 9 on the MetS, 4 on T2DM and 10 on CVD (Figure 1).

\section{Results}

\subsection{Characteristics of Study Sample}

Most of the studies were from Mediterranean countries, specifically in Southern Europe, except for three conducted in the USA [32-34], two in Canada [35,36] and three in European countries outside the Mediterranean area [37-39]. Most had been conducted in both men and women, three studies only included females [35,38,40] and three involved children or adolescents [41-43]. All the studies were published between 2003 and 2013. The health status of the subjects varied among studies, but in the current review only includes studies on primary prevention. 
Figure 1. Flow-chart of the selection process. Abbreviations are as follows: METS, metabolic syndrome; T2DM, type 2 diabetes mellitus; CVD, cardiovascular disease.

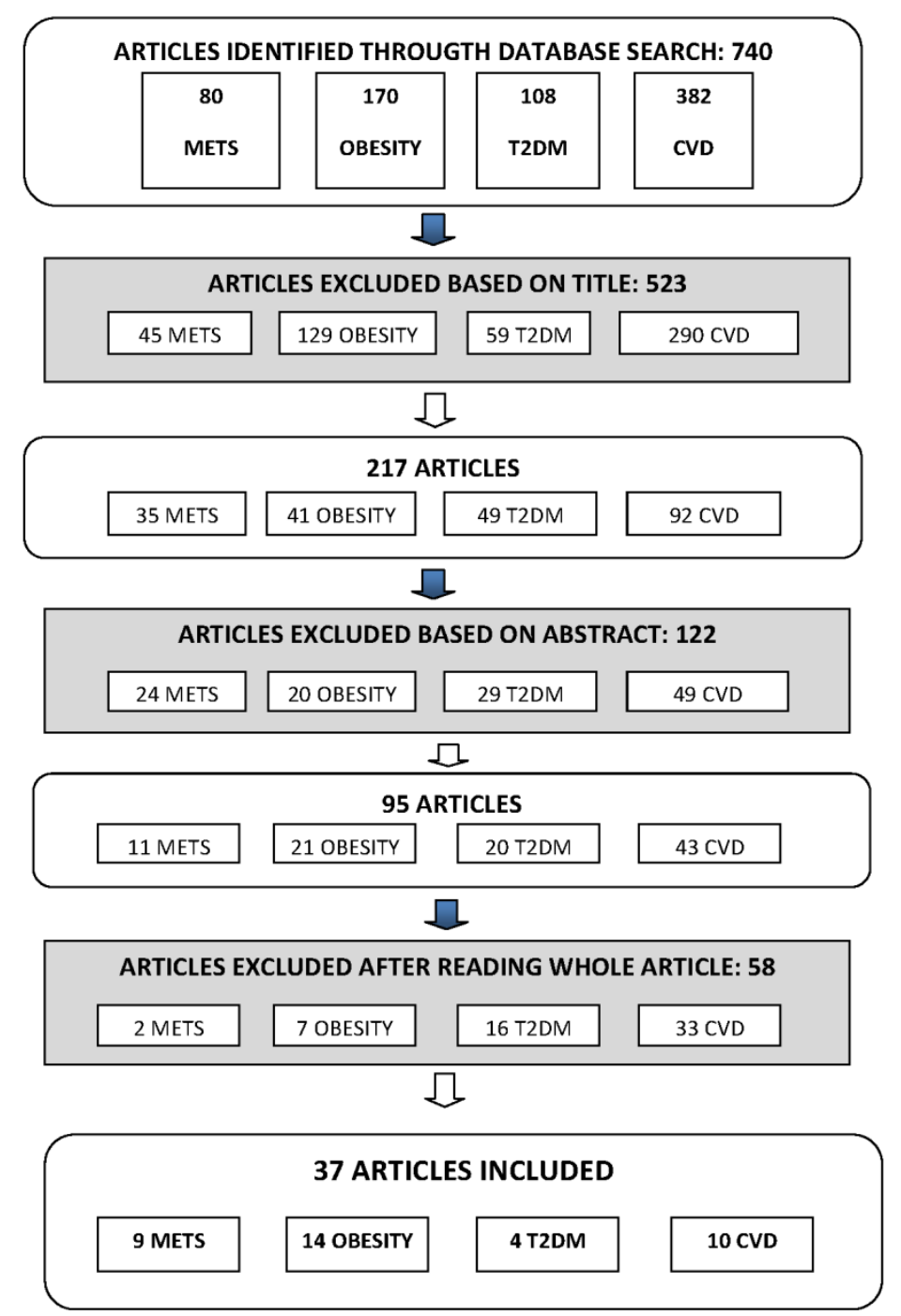

The criteria of quality considered for the studies selected were the sample size, follow-up period and adjustment for potential confounders (demographic, anthropometric and traditional cardiovascular risk factors). Information regarding the methodology and weight-related results for these studies are summarized in Table 1. The sample sizes varied from 77 [35] and 497,308 [37] individuals. The potential confounders considered in the statistical analysis varied among studies, but the most common were age, sex, energy intake, smoking, physical activity and educational level, and also body mass index, marital status, family history of disease or pharmacological treatment.

To assess adherence to the MedDiet pattern, most of the studies included used the Mediterranean diet score established in 2003 by Trichopolou et al. [44,45], albeit with some modifications in many studies (detailed on Table 1). In addition, a considerable number of studies established their own score, for instance in the PREDIMED trial [46] the score for adherence to the MedDiet is based on a 14-item index. 
Table 1. Characteristics of the studies included evaluating the relationship between the Mediterranean Diet and cardiodiabesity.

\begin{tabular}{|c|c|c|c|c|c|c|c|c|c|}
\hline $\begin{array}{c}\text { Author/Year of } \\
\text { Publication } \\
\end{array}$ & Country & Gender & $\begin{array}{c}\text { Age } \\
\text { (Years) }\end{array}$ & $\begin{array}{c}\text { Follow-Up } \\
\text { (Years) }\end{array}$ & $N$ & Components in the MD Index & $\begin{array}{c}\text { Main } \\
\text { Outcome }\end{array}$ & Results & Confounders \\
\hline $\begin{array}{l}\text { Panagiotakos et al. } \\
2005 \text { [47] }\end{array}$ & Greece & $\begin{array}{l}\text { T1514 } \\
+1528\end{array}$ & $\begin{array}{l}\text { ग20-87; } \\
+20-89\end{array}$ & 1.25 & 3042 & $\begin{array}{l}\text { Score based on dietary pyramid [48]: } \\
\text { (d) non refined cereals and products, } \\
\text { fruits, olive oil, dairy products, wine; } \\
\text { (w) fish, poultry, potatoes, olives, } \\
\text { legumes, nuts, eggs, sweets; } \\
\text { (m) red meat }\end{array}$ & $\mathrm{T} 2 \mathrm{DM}$ & $\begin{array}{l}\text { With } 10 \text { P increase score: } \\
\text { OR } 0.79(95 \% \text { CI } 0.65-0.94), p<0.05) \text {; } \\
\text { ARR } 1.3 \%(5.6 \%-4.3 \%) \\
\text { RRR } 23.2 \%[(5.6 \%-4.3 \%) / 5.6 \%]\end{array}$ & $\begin{array}{l}\text { Age, sex, BMI, WC, hc, } \\
\text { education, financial } \\
\text { status, smoking, family } \\
\text { history of T2DM }\end{array}$ \\
\hline $\begin{array}{l}\text { Martínez-Glez et al. } \\
\qquad 2008 \text { [49] }\end{array}$ & Spain & $\begin{array}{l}\text { Men and } \\
\text { women }\end{array}$ & $20-90$ & 4.4 & 13,380 & $\begin{array}{l}\text { MDS [45]: (+) fatty acid ratio, } \\
\text { legumes, grains, fruits, vegetables, } \\
\text { fish, wine; }(-) \text { dairy products or meat }\end{array}$ & T2DM & $\begin{array}{l}\text { OR } 0.41(95 \% \text { CI 0.19-0.87) (score 3-6); } \\
\text { OR } 0.17(0.04-0.75) \text { (score 7-9). } \\
\text { With } 2 \text { P increase score } \\
\text { RR 0.65, }(0.44-0.95), p=0.04\end{array}$ & $\begin{array}{l}\text { Sex, age, years of } \\
\text { university education, TEI, } \\
\text { BMI, PA, sedentary } \\
\text { habits, smoking, family } \\
\text { history of T2DM, and } \\
\text { personal history of HT }\end{array}$ \\
\hline $\begin{array}{l}\text { Salas-Salvadó et al. } \\
\qquad 2011[50]\end{array}$ & Spain & $\begin{array}{l}\text { Men and } \\
\text { women }\end{array}$ & $55-80$ & 4 & 418 & $\begin{array}{l}\text { PREDIMED score } 14 \text { items [51]: } \\
\text { olive oil, vegetables, fruit, fatty acid } \\
\text { ratio, legumes, wine, fish, nuts; } \\
\text { (-) sweets and carbonated beverages, } \\
\text { red meat }\end{array}$ & $\mathrm{T} 2 \mathrm{DM}$ & $\begin{array}{l}\text { T2DM incidence MedDiet VOO } 10.1 \% \\
(95 \% \text { CI } 5.1-15.1) \text {, MedDiet nuts } 11.0 \% \\
(5.9-16.1) \text {, control group } 17.9 \%(11.4-24.4) \text {. } \\
\text { MedDiet VOO OR } 0.49(0.25-0.97) \text { and nuts } \\
\text { groups } 0.48(0.24-0.96) \text {. } \\
\text { DM incidence was reduced } 52 \%(27-86)\end{array}$ & $\begin{array}{l}\text { Age, sex, TEI, BMI, WC, } \\
\text { PA, smoking status, } \\
\text { fasting serum glucose, } \\
\text { use of lipid lowering } \\
\text { drugs, MedDiet score, } \\
\text { and weight change during } \\
\text { the study }\end{array}$ \\
\hline
\end{tabular}


Table 1. Cont.

\begin{tabular}{|c|c|c|c|c|c|c|c|c|c|}
\hline $\begin{array}{l}\text { Author/Year } \\
\text { of Publication }\end{array}$ & Country & Gender & $\begin{array}{c}\text { Age } \\
\text { (Years) }\end{array}$ & $\begin{array}{c}\text { Follow-Up } \\
\text { (Years) }\end{array}$ & $N$ & Components in the MD Index & $\begin{array}{c}\text { Main } \\
\text { Outcome }\end{array}$ & Results & Confounders \\
\hline $\begin{array}{c}\text { Abiemo et al. } \\
2013 \text { [33] }\end{array}$ & USA & $\begin{array}{l}\text { Men and } \\
\text { women }\end{array}$ & $45-84$ & 6 & 5390 & $\begin{array}{l}\text { Score was created }[45] \text { : vegetables, } \\
\text { whole grains, nuts, legumes, fruits, } \\
\text { MUFA/SFA, red and processed meat, } \\
\text { dairy products, fish, } \mathrm{OH}\end{array}$ & $\mathrm{T} 2 \mathrm{DM}$ & $\begin{array}{l}\text { MeDiet score: lower baseline mean insulin levels, } \\
\text { Q1: } 5.8(95 \% \text { CI } 5.6-6.0) \mu \mathrm{mol} / \mathrm{L} ; \\
\text { Q5: } 4.8(95 \% \text { CI } 4.6-5.0) \mu \mathrm{mol} / \mathrm{L} ; p<0 \cdot 0001 . \\
\text { MedDiet not significantly related risk of T2DM } \\
p=0.64\end{array}$ & $\begin{array}{l}\text { Demographic, } \\
\text { physiological and } \\
\text { behavioral characteristics }\end{array}$ \\
\hline $\begin{array}{l}\text { Panagiotakos et al. } \\
2004 \text { [52] }\end{array}$ & Greece & $\begin{array}{l}\text { Men and } \\
\text { women }\end{array}$ & $>18$ & l & 2282 & $\begin{array}{l}\text { Score created [53]: (d) cereals and } \\
\text { products, vegetables, fruits, olive oil, } \\
\text { dairy products; (w) fish, poultry, } \\
\text { olives, legumes, nuts, potatoes, eggs, } \\
\text { sweets; (m) red meat; (+m) wine and } \\
\text { high MUFA/SFA ratio }\end{array}$ & MetS & OD 0.81 (95\% CI, 0.68-0.976) & $\begin{array}{l}\text { Age, sex, PA, education, } \\
\text { smoking, BMI, diet in the } \\
\text { past } 12 \text { months, TEI }\end{array}$ \\
\hline $\begin{array}{l}\text { Álvarez-León et al. } \\
2006 \text { [54] }\end{array}$ & Spain & $\begin{array}{l}\text { Men and } \\
\text { women }\end{array}$ & $>18$ & l & 578 & $\begin{array}{l}\text { 10-Item score created [55]: cereal, } \\
\text { fruits, vegetables, legumes, fish, nuts, } \\
\text { alcohol intake from red wine, } \\
\text { MUFA/SFA and dairy products }\end{array}$ & MetS & $\begin{array}{l}\text { MD adherence not related to MetS prevalence; } \\
\text { T2 OR } 1.39 \text { (95\% CI, 0.75-2.59); } \\
\text { T3 OR } 1.37 \text { (95\% CI, 0.76-2.46) }\end{array}$ & $\begin{array}{l}\text { Age, sex, PA, education, } \\
\text { smoking, BMI, diet in the } \\
\text { past } 12 \text { months, TEI }\end{array}$ \\
\hline $\begin{array}{c}\text { Tortosa et al. } \\
2007 \text { [56] }\end{array}$ & Spain & $\begin{array}{l}\text { Men and } \\
\text { women }\end{array}$ & $20-90$ & 6 & 2563 & $\begin{array}{l}\text { MDS [44]: }(+) \text { fatty acid ratio, } \\
\text { legumes, grains, fruits, vegetables, } \\
\text { fish, wine; }(-) \text { dairy products or meat. }\end{array}$ & MetS & $\begin{array}{l}\text { MFP }(95 \% \text { CI) Age \& sex adjusted } \\
\text { score 3-5 OR } 0.76(0.40-1.45) \\
\text { score 6-9 OR } 0.18(0.06-0.56) p=0.006 ; \\
\text { Multivariate-adjusted } \\
\text { score 3-5 OR } 0.80(0.42-1.54) \\
\text { score 6-9 OR } 0.20(0.06-0.63)\end{array}$ & $\begin{array}{l}\text { Age, sex, PA, } \\
\text { smoking, TEI }\end{array}$ \\
\hline
\end{tabular}


Table 1. Cont

\begin{tabular}{|c|c|c|c|c|c|c|c|c|c|}
\hline $\begin{array}{l}\text { Author/year } \\
\text { of Publication }\end{array}$ & Country & Gender & $\begin{array}{c}\text { Age } \\
\text { (Years) }\end{array}$ & $\begin{array}{c}\text { Follow-Up } \\
\text { (Years) }\end{array}$ & $N$ & Components in the MD Index & $\begin{array}{c}\text { Main } \\
\text { Outcome }\end{array}$ & Results & Confounders \\
\hline $\begin{array}{l}\text { Salas-Salvadó et al. } \\
\qquad 2008 \text { [57] }\end{array}$ & Spain & $\begin{array}{l}\text { Men and } \\
\text { women }\end{array}$ & $\begin{array}{l}\text { क5 } 55-80 \\
+60-80\end{array}$ & 1 & 1224 & $\begin{array}{l}\text { PREDIMED score } 14 \text { items [51]: } \\
\text { olive oil, vegetables, fruit, fatty } \\
\text { acid ratio, legumes, wine, fish, } \\
\text { nuts; (-) sweets and carbonated } \\
\text { beverages, meat }\end{array}$ & MetS & $\begin{array}{l}\text { MedDiet VOO group OR } 1.3(95 \% \text { CI } 0.8-2.1) \\
\text { MedDiet nuts group OR } 1.7(1.1-2.6)\end{array}$ & $\begin{array}{l}\text { Sex, age, baseline obesity } \\
\text { status, and weight changes }\end{array}$ \\
\hline $\begin{array}{l}\text { Babio et al. } \\
2009[58]\end{array}$ & Spain & $\begin{array}{l}\text { Men and } \\
\text { women }\end{array}$ & Mean 67 & l & 808 & $\begin{array}{l}\text { 14-P questionnaire [59]: olive oil, } \\
\text { vegetables, fruit, fatty acid ratio, } \\
\text { legumes, wine, fish, nuts; }(-) \text { sweets } \\
\text { and carbonated beverages, meat }\end{array}$ & MetS & $\uparrow \mathrm{MD}$ score OR $0.44(95 \% \mathrm{CI}, 0.27-0.70)$ & $\begin{array}{l}\text { Age, sex, PA, } \\
\text { smoking, TEI }\end{array}$ \\
\hline $\begin{array}{c}\text { Bibiloni et al. } \\
2011[43]\end{array}$ & Spain & $\begin{array}{l}\text { Men and } \\
\text { women }\end{array}$ & $12-17$ & l & 362 & $\begin{array}{l}\text { Score created [45]: high MUFA/SFA } \\
\text { ratio; }(+\mathrm{m}) \mathrm{OH} \text {, high legume, cereals } \\
\text { and roots, fruit, vegetables, and fish } \\
\text { consumption, and low meat and milk } \\
\text { consumption }\end{array}$ & MetS & $\begin{array}{l}\text { Q2 MD 6.9\%; Q4 5.9\%; } \\
\text { Glucose levels } 0.89 \text { (95\% CI 0.40-1.90), } \\
\text { triglyceride levels } 0.72 \text { (95\% CI 0.31-1.77) and } \\
\text { HDL-cholesterol levels } 0.43 \text { (95\% CI } 0.19-0.99)\end{array}$ & $\begin{array}{l}\text { Sex, age, parental } \\
\text { educational level, } \\
\text { parental socioeconomic } \\
\text { status and PA }\end{array}$ \\
\hline $\begin{array}{l}\text { Rumawas et al. } \\
\qquad 2009[34]\end{array}$ & EEUU & $\begin{array}{l}\text { Men and } \\
\text { women }\end{array}$ & Mean 54 & 7 & 2730 & $\begin{array}{l}\text { Score created based on MSDPS [60]: } \\
\text { whole-grain cereals, fruit, vegetables, } \\
\text { dairy, wine, fish, poultry, } \\
\text { olives/legumes/nuts, potatoes, eggs, } \\
\text { sweets, meat, and olive oil }\end{array}$ & MetS & $\begin{array}{l}\text { Q5 MSDPS } \downarrow \text { incidence MetS }(38.5 \% \text { vs. 30.1\%; } \\
p=0.01)(95 \% \text { CI) HOMA-IR Q5 3.16 }(3.03-3.30) \\
p=0.02 \text {; fasting glucose } 97.1(96.3-98.0) p=0.03 ; \\
\text { waist circumference } 97.1(96.7-97.6) p<0.001 ; \\
\text { triglycerides } 103(99-107) p<0.001 ; \\
\text { HDL-cholesterol } 54.0(53.1-55.0) p=0.02\end{array}$ & $\begin{array}{l}\text { Height, weight, BMI, age, } \\
\text { sex, smoking, multivitamin } \\
\text { use, ERT, PA, TEI }\end{array}$ \\
\hline
\end{tabular}


Table 1. Cont.

\begin{tabular}{|c|c|c|c|c|c|c|c|c|c|}
\hline $\begin{array}{l}\text { Author/Year } \\
\text { of Publication }\end{array}$ & Country & Gender & $\begin{array}{c}\text { Age } \\
\text { (Years) }\end{array}$ & $\begin{array}{c}\text { Follow-up } \\
\text { (Years) }\end{array}$ & $N$ & Components in the MD Index & $\begin{array}{c}\text { Main } \\
\text { Outcome }\end{array}$ & Results & Confounders \\
\hline $\begin{array}{l}\text { Paletas et al. } \\
2010[61]\end{array}$ & Greece & $\begin{array}{l}\text { Men and } \\
\text { women }\end{array}$ & $44.4 \pm 13.35$ & / & 226 & $\begin{array}{l}\text { MDS [45]: }(+) \text { fatty acid ratio, } \\
\text { legumes, grains, fruits, vegetables, } \\
\text { fish, wine; }(-) \text { dairy products } \\
\text { or meat }\end{array}$ & MetS & $\begin{array}{l}\text { MetS components } \uparrow \text { non-MD: MetS prevalence } \\
27.3 \% \text { MedDiet group, } 60.2 \% \text { HF group, and } \\
\text { HC group } 69.2 \% p=0.006\end{array}$ & Sex, BMI, TEI \\
\hline $\begin{array}{l}\text { Kesse-Guyot et al. } \\
\quad 2013 \text { [62] }\end{array}$ & France & $\begin{array}{l}\text { Men and } \\
\text { women }\end{array}$ & $>18$ & 6 & 3232 & $\begin{array}{l}\text { MDS [45], MSDPS [60] and } \\
\text { MED [63] }\end{array}$ & MetS & $\begin{array}{l}\downarrow \text { MetS: high MED OR } 0.47(95 \% \text { CI } 0.32-0.69) \\
p=0.001 ; \text { high MDS OR } 0.50(95 \% \text { CI } 0.32-0.77) \\
p=0.03\end{array}$ & $\begin{array}{l}\text { Age, gender, education } \\
\text { level, smoking status, } \\
\text { PA, TEI, antidiabetic, } \\
\text { antihypertensive and lipid } \\
\text { lowering medications }\end{array}$ \\
\hline $\begin{array}{l}\text { Goulet et al. } \\
2003 \text { [35] }\end{array}$ & Canada & Women & $30-65$ & 0.25 & 77 & $\begin{array}{l}\text { MD: }(+) \text { cereals, fruit, vegetables, } \\
\text { legumes, nuts and seeds, fish, olive } \\
\text { oil, wine; }(-) \text { dairy products, } \\
\text { chicken, eggs, sweets, red meat }\end{array}$ & OW and $\mathrm{OB}$ & $\begin{array}{l}\uparrow \mathrm{MD}, \downarrow \text { BMI (mean week 0: } 25.8 \pm 3.9 \mathrm{~kg} / \mathrm{m}^{2} \text { to } \\
\left.\text { mean week } 325.6 \pm 3.8 \mathrm{~kg} / \mathrm{m}^{2}\right) p<0.01 ; \text { adh less } \\
\text { weight }(\mathrm{m} 0: 67.7 \pm 11.9 \mathrm{~kg} \text { to } \mathrm{m} 3: 67.3 \pm 11 \mathrm{~kg}) \\
p<0.01\end{array}$ & \\
\hline $\begin{array}{l}\text { Schröder et al. } \\
2004[64]\end{array}$ & Spain & $\begin{array}{l}\text { ô1403 } \\
\text { +1 } 1468\end{array}$ & $25-74$ & 2 & 3162 & $\begin{array}{l}\text { MDS [44]: }(+) \text { cereals, vegetables, } \\
\text { fruit, legumes, nuts, fish, red wine; } \\
(-) \text { meat, high fat dairy products }\end{array}$ & OB & $\begin{array}{l}\uparrow \mathrm{MD}, \downarrow \text { OB OR } 0.61 p=0.01 \\
\text { With } 5 \text {-P MD, less BMI } \delta^{-}-0.43 \mathrm{~kg} / \mathrm{m}^{2} p=0.030 \\
\text { ㅇ }-0.68 \mathrm{~kg} / \mathrm{m}^{2} p=0.007\end{array}$ & $\begin{array}{l}\text { Age, TEI, educational } \\
\text { level, smoking, LTPA, } \\
\text { smoking and alcohol } \\
\text { consumption }\end{array}$ \\
\hline
\end{tabular}


Table 1. Cont.

\begin{tabular}{|c|c|c|c|c|c|c|c|c|c|}
\hline $\begin{array}{l}\text { Author/Year } \\
\text { of Publication }\end{array}$ & Country & Gender & $\begin{array}{c}\text { Age } \\
\text { (Years) } \\
\end{array}$ & $\begin{array}{c}\text { Follow-Up } \\
\text { (Years) }\end{array}$ & $N$ & Components in the MD Index & $\begin{array}{c}\text { Main } \\
\text { Outcome }\end{array}$ & Results & Confounders \\
\hline $\begin{array}{l}\text { Trichopoulou et al. } \\
\qquad 2005[65]\end{array}$ & Greece & $\begin{array}{l}\text { İ9612 } \\
+13,985\end{array}$ & $20-86$ & / & 23,597 & $\begin{array}{l}\text { MDS [44]: }(+) \text { cereals, vegetables, } \\
\text { fruit and nuts, legumes, fish, } \\
\text { MUFA/SFA; }(+\mathrm{m}) \mathrm{OH} ;(-) \text { meat } \\
\text { and dairy products }\end{array}$ & OB & 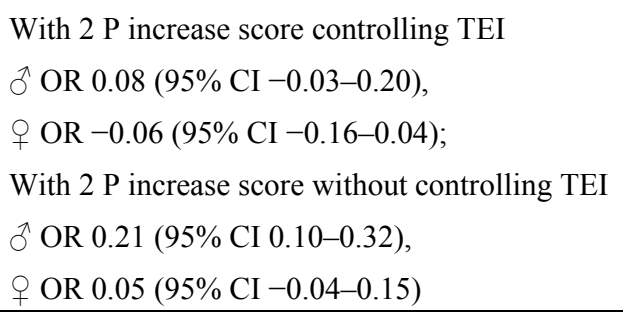 & $\begin{array}{l}\text { Age, years of schooling, } \\
\text { smoking, PA, TEI }\end{array}$ \\
\hline $\begin{array}{c}\text { Shubair et al. } \\
2005 \text { [36] }\end{array}$ & Canada & $\begin{array}{l}7265 \\
+494\end{array}$ & $18-65$ & / & 759 & $\begin{array}{l}\text { MDP score created: }(+) \text { fruit, } \\
\text { vegetables, olive oil and garlic, } \\
\text { fish and shellfish; }(-) \text { meats and } \\
\text { poultry, high SFA, foods high in } \\
\text { added sugar and low nutrients }\end{array}$ & OB & $\begin{array}{l}\uparrow \mathrm{MD}, \downarrow \mathrm{BMI}(\beta \text {-coefficient }-0.186) \\
p=0.027\end{array}$ & $\begin{array}{l}\text { Sex, education, income and } \\
\text { marital status }\end{array}$ \\
\hline $\begin{array}{l}\text { Panagiotakos et al. } \\
\qquad 2006[66]\end{array}$ & Greece & $\begin{array}{l}\uparrow 1514 \\
+1528\end{array}$ & $18-89$ & l & 3042 & $\begin{array}{l}\text { MDS [48]: wholegrain cereals, } \\
\text { vegetables, fruit, legumes, fish, } \\
\text { olive oil, dairy products, chicken, } \\
\text { nuts and seeds, olives, potatoes, } \\
\text { eggs, sweets, meat, MUFA/SFA }\end{array}$ & $\begin{array}{l}\text { OW and } \\
\text { OB }\end{array}$ & $\begin{array}{l}\text { With } 5 \text { P increased score OR } 0.49 \\
(95 \% \text { CI } 0.42-0.56)(\mathrm{OB} \& \mathrm{OW}) \\
\uparrow \mathrm{MD}, \downarrow \mathrm{BMI}(-4 \mathrm{~kg}) p=0.001\end{array}$ & $\begin{array}{l}\text { Age, sex, PA, metabolism, } \\
\text { educational level, smoking status }\end{array}$ \\
\hline $\begin{array}{c}\text { Mendez et al. } \\
2006[67]\end{array}$ & Spain & $\begin{array}{l}\text { O1 } 10,589 \\
+17,238\end{array}$ & $29-65$ & 3.3 & 27,827 & $\begin{array}{l}\text { MDS [65]: }(+) \text { cereals, vegetables, } \\
\text { fruit, legumes, fish, MUFA/SFA; } \\
(+\mathrm{m}) \mathrm{OH} ;(-) \text { meat }\end{array}$ & OB & 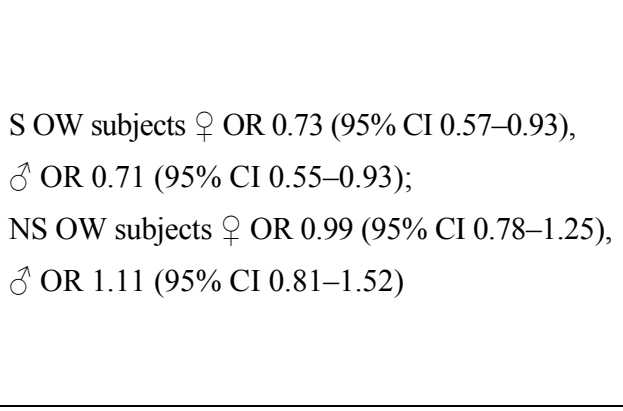 & $\begin{array}{l}\text { Age, special diets related to } \\
\text { obesity or related disorders, } \\
\text { categorical activity index, } \\
\text { education, center, height, parity, } \\
\text { smoking status, winter season, } \\
\text { follow-up time, health status and } \\
\text { changes in lifestyle or health } \\
\text { during follow-up }\end{array}$ \\
\hline
\end{tabular}


Table 1. Cont.

\begin{tabular}{|c|c|c|c|c|c|c|c|c|c|}
\hline $\begin{array}{l}\text { Author/Year } \\
\text { of Publication }\end{array}$ & Country & Gender & $\begin{array}{c}\text { Age } \\
\text { (Years) }\end{array}$ & $\begin{array}{c}\text { Follow-Up } \\
\text { (Years) }\end{array}$ & $N$ & Components in the MD Index & Main Outcome & Results & Confounders \\
\hline $\begin{array}{l}\text { Andreoli et al. } \\
2008[40]\end{array}$ & Italy & Women & $25-70$ & 0.33 & 47 & $\begin{array}{l}\text { MD moderately hypo-caloric: } \\
(+) \text { vegetables, fruit, pasta, bread, } \\
\text { legumes, fish, olive oil, red wine, } \\
\text { meat, dairy products \& PA program }\end{array}$ & $\begin{array}{l}\text { Cardiovascular } \\
\text { disease risk factors } \\
\text { in obese women }\end{array}$ & $\begin{array}{l}\uparrow \mathrm{MD}, \downarrow \text { weight }(\mathrm{m} 080.4 \pm 15.8 \mathrm{~kg} \text { to } \\
\mathrm{m} 475.2 \pm 14.7 \mathrm{~kg}) p<0.001 ; \\
\uparrow \mathrm{MD}, \downarrow \mathrm{BMI}(\mathrm{m} 030.7 \pm 6.0 \mathrm{~kg} \text { to } \\
\mathrm{m} 428.7 \pm 5.6 \mathrm{~kg}) p<0.001\end{array}$ & \\
\hline $\begin{array}{l}\text { Panagiotakos et al. } \\
\quad 2007[68]\end{array}$ & Cyprus & $\begin{array}{l}\text { †53 } \\
+97\end{array}$ & $65-100$ & / & 150 & $\begin{array}{l}\text { MDS: wholegrain cereals, vegetables, } \\
\text { fruit, legumes, fish, olive oil, dairy } \\
\text { products, chicken, nuts, seeds, olives, } \\
\text { potatoes, eggs, sweets, meat }\end{array}$ & OB & $\begin{array}{l}\text { With } 10 \mathrm{P} \text { increased score } \mathrm{OR} 0.83 \\
(95 \% \mathrm{CI}-0.25-0.12) p<0.001 \\
\text { (having one additional risk factor); } \\
\text { With } 10 \mathrm{P} \text { increased score } 12 \% \text { lower } \\
\text { OR OB } p=0.001\end{array}$ & $\begin{array}{l}\text { Age, sex, smoking } \\
\text { habits, PA }\end{array}$ \\
\hline $\begin{array}{l}\text { Romaguera et al. } \\
\quad 2009 \text { [37] }\end{array}$ & $\begin{array}{c}10 \text { European } \\
\text { countries }\end{array}$ & $\begin{array}{l}\text { Men and } \\
\text { women }\end{array}$ & $25-70$ & 8 & 497,308 & $\begin{array}{l}\text { mMDS [44]: }(+) \text { vegetables, legumes, } \\
\text { fruits, nuts, cereals, fish and seafood, } \\
\text { MUFA/SFA; }(+\mathrm{m}) \mathrm{OH} ;(-) \text { meat, } \\
\text { dairy products }\end{array}$ & OB & $\begin{array}{l}\uparrow \mathrm{MD}, \downarrow \text { WC, for a given BMI } \\
\text { o } 20.09 \text { (95\% CI 20.14-20.04), } \\
\text { † } 20.06 \text { (95\% CI 20.10-20.01); } \\
\text { Northern European countries } \\
\text { o } 20.20 \text { (95\% CI 20.23-20.17), } \\
\text { † } 20.17 \text { (95\% CI 20.21-20.13) }\end{array}$ & $\begin{array}{l}\text { Age, educational level } \\
\text { (categorical), PA, } \\
\text { smoking status, height, } \\
\text { menopausal status }\end{array}$ \\
\hline $\begin{array}{c}\text { Beunza et al. } \\
2010 \text { [69] }\end{array}$ & Spain & $\begin{array}{l}\text { Men and } \\
\text { women }\end{array}$ & Mean 38 & $5.7 \pm 2.2$ & 10,376 & $\operatorname{MDS}[45]$ & $\mathrm{OW}$ and $\mathrm{OB}$ & $\begin{array}{l}\uparrow \mathrm{MD}, \downarrow \text { weight } 20.059 \mathrm{~kg} / \text { year } \\
95 \% \text { CI } 20.111-20.008 \mathrm{~kg} / \text { year, } \\
p=0.02 \text {; lowest risk weight gain } \\
\text { 4-year } 0.76(95 \% \text { CI } 0.64-0.90)\end{array}$ & $\begin{array}{l}\text { Age, sex, baseline BMI, } \\
\text { PA, sedentary behaviors, } \\
\text { smoking, between-meals } \\
\text { snacking, TEI }\end{array}$ \\
\hline
\end{tabular}


Table 1. Cont

\begin{tabular}{|c|c|c|c|c|c|c|c|c|c|}
\hline $\begin{array}{l}\text { Author/Year } \\
\text { of Publication }\end{array}$ & Country & Gender & $\begin{array}{c}\text { Age } \\
\text { (Years) }\end{array}$ & $\begin{array}{c}\text { Follow-Up } \\
\text { (Years) }\end{array}$ & $N$ & $\begin{array}{l}\text { Components in the } \\
\text { MD Index }\end{array}$ & $\begin{array}{c}\text { Main } \\
\text { Outcome }\end{array}$ & Results & Confounders \\
\hline $\begin{array}{l}\text { Romaguera et al. } \\
\quad 2010[70]\end{array}$ & $\begin{array}{l}10 \text { European } \\
\text { countries }\end{array}$ & $\begin{array}{l}\text { Men and } \\
\text { women }\end{array}$ & $25-70$ & 5 & 373,803 & $\begin{array}{l}\text { rMED [71]: }(+) \text { vegetables, } \\
\text { legumes, fruit, nuts, cereals, } \\
\text { fish and seafood, olive oil; } \\
(+\mathrm{m}) \mathrm{OH} ;(-) \text { meat, dairy } \\
\text { products }\end{array}$ & OW \& OB & $\begin{array}{l}\uparrow \mathrm{rMED}, \downarrow \text { weight gain } 5 \text {-year } 20.16 \mathrm{~kg} \\
(95 \% \mathrm{CI} 20.24,20.07 \mathrm{~kg}) \text {; less likely to } \\
\text { OW or OB } 10 \%(95 \% \text { CI } 4 \%-18 \%)\end{array}$ & $\begin{array}{l}\text { Sex, age, baseline BMI, } \\
\text { follow-up, educational level, } \\
\text { PA, smoking status, } \\
\text { menopausal status, TEI, } \\
\text { misreporting of TEI }\end{array}$ \\
\hline $\begin{array}{l}\text { Lazarou et al. } \\
2010[41]\end{array}$ & Cyprus & $\begin{array}{l}\text { Men and } \\
\text { women }\end{array}$ & $\begin{array}{c}\text { Mean } \\
10.7 \pm 0.98\end{array}$ & l & 1140 & KIDMED [72] & OB & $\begin{array}{l}\uparrow \text { KIDMED, } \downarrow \text { likely to OW/OB } 80 \% \\
(95 \% \text { CI } 0.041-0.976)\end{array}$ & $\begin{array}{l}\text { Age, gender, parental obesity } \\
\text { status, parental educational } \\
\text { level, dietary beliefs and } \\
\text { behaviors }\end{array}$ \\
\hline $\begin{array}{c}\text { Farajian et al. } \\
2011[42]\end{array}$ & Greece & Children & $10-12$ & l & 4786 & KIDMED [72] & OW \& OB & $\begin{array}{l}\text { NS NW (score mean } 3.70 \pm 2.26) \& \mathrm{OW} / \mathrm{OB} \\
\text { (score mean } 3.62 \pm 2.26), t \text {-test } 1.08, p=0.28\end{array}$ & $\begin{array}{l}\text { Age, BMI, waist, body } \\
\text { fat mass }\end{array}$ \\
\hline $\begin{array}{l}\text { Martínez-Glez et al. } \\
\qquad 2012 \text { [73] }\end{array}$ & Spain & $\begin{array}{l}\text { Men and } \\
\text { women; } \\
57 \% \text { }\end{array}$ & $55-80$ & l & 7447 & PREDIMED [51] & OB & 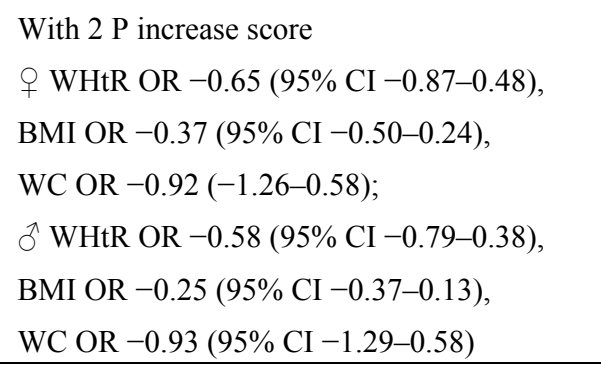 & $\begin{array}{l}\text { Age, smoking, diabetes } \\
\text { status, HT status, PA, } \\
\text { educational level, marital } \\
\text { status, center, TEI }\end{array}$ \\
\hline $\begin{array}{l}\text { Trichopoulou et al. } \\
\qquad 2003 \text { [45] }\end{array}$ & Greece & $\begin{array}{l}\text { Men and } \\
\text { women }\end{array}$ & $20-86$ & Median 3.7 & 22,043 & $\begin{array}{l}9 \text { Unit MDS [44]: } \\
(+) \text { vegetables, legumes, } \\
\text { fruits, nuts, cereals, fish; } \\
(+\mathrm{m}) \mathrm{OH} ;(-) \text { meat, poultry, } \\
\text { dairy products }\end{array}$ & $\begin{array}{c}\text { Fatal CHD } \\
\text { events }\end{array}$ & $\begin{array}{l}\uparrow \mathrm{MD}, \downarrow \text { CHD mortality HR } 0.67 \\
(95 \% \text { CI } 0.47-0.94)\end{array}$ & $\begin{array}{l}\text { Sex, age, years of education, } \\
\text { smoking status, WtHR, BMI, } \\
\text { TEI, energy-expenditure score }\end{array}$ \\
\hline
\end{tabular}


Table 1. Cont.

\begin{tabular}{|c|c|c|c|c|c|c|c|c|c|}
\hline $\begin{array}{l}\text { Author/Year } \\
\text { of Publication }\end{array}$ & Country & Gender & $\begin{array}{c}\text { Age } \\
\text { (Years) }\end{array}$ & $\begin{array}{c}\text { Follow-Up } \\
\text { (Years) }\end{array}$ & $N$ & Components in the MD Index & $\begin{array}{c}\text { Main } \\
\text { Outcome }\end{array}$ & Results & Confounders \\
\hline $\begin{array}{c}\text { Knoops et al. } \\
2004 \text { [39] }\end{array}$ & $\begin{array}{l}11 \text { European } \\
\text { Countries }\end{array}$ & $\begin{array}{l}\text { Men and } \\
\text { women }\end{array}$ & $70-90$ & 12 & 2339 & $\begin{array}{l}\text { mMD [45]: MUFA/SFA, } \\
\text { legumes, nuts, seeds, grains, } \\
\text { fruit, vegetables and potatoes, } \\
\text { meat, dairy products, fish }\end{array}$ & $\begin{array}{c}\text { All-cause and } \\
\text { cause specific } \\
\text { mortality }\end{array}$ & $\begin{array}{l}\uparrow \text { MD score, } \downarrow \text { mortality: all causes } \\
0.77(0.68-0.88), \text { CHD } 0.61(0.43-0.88) \\
\text { CVD } 0.71(0.58-0.88)(95 \% \mathrm{CI})\end{array}$ & $\begin{array}{l}\text { Other diet and lifestyle } \\
\text { factors, sex, age at baseline, } \\
\text { BMI, and study population } \\
\text { (SENECA vs. FINE). }\end{array}$ \\
\hline $\begin{array}{l}\text { Panagiotakos et al. } \\
2007[74]\end{array}$ & Greece & $\begin{array}{l}\text { Men and } \\
\text { women }\end{array}$ & $18-89$ & & 3042 & $\begin{array}{l}\text { Own score [48]: }(+) \text { non-refined } \\
\text { cereals, fruit, vegetables, } \\
\text { legumes, potatoes, fish, olive oil; } \\
(-) \text { meat, poultry, full fat dairy } \\
\text { products; }(+\mathrm{m}) \mathrm{OH}\end{array}$ & $\begin{array}{l}\text { CVD risk } \\
\text { factors }\end{array}$ & $\begin{array}{l}\text { With } 10 \mathrm{P} \text { increase score, } 10 \text {-year follow up } \\
4 \% \text { lower CHD risk }( \pm 0.1 \%, p<0.001) \text {; } \\
\uparrow \mathrm{MD}, \downarrow 10 \text {-year follow up CHD risk } \\
\text { (rho }-0.7, p<0.001 \text { ) }\end{array}$ & $\begin{array}{l}\text { No assessment for } \\
\text { potential confounding since } \\
\text { all analyses were unadjusted }\end{array}$ \\
\hline $\begin{array}{c}\text { Buckland et al. } \\
2009 \text { [71] }\end{array}$ & Spain & $\begin{array}{l}\text { Men and } \\
\text { women }\end{array}$ & $29-69$ & Mean 10.4 & 41,078 & $\begin{array}{l}\text { r-MED [45]: }(+) \text { fruits, nuts, } \\
\text { seeds, vegetables (no potatoes), } \\
\text { legumes, cereals, fresh fish, } \\
\text { olive oil; (-) meat, dairy products }\end{array}$ & CHD events & $\begin{array}{l}\uparrow \text { MD score, } \downarrow \text { CHD risk, } \\
Z 1 / 40.60 \text { (95\% CI } 0.47-0.77) ; \\
\text { with } 1 \text { P increase score, } 6 \% \text { reduced risk } \\
\text { CHD }(95 \% \text { CI } 0.91-0.97)\end{array}$ & $\begin{array}{l}\text { Age, BMI, educational } \\
\text { level, smoking status, } \\
\text { PA, TEI, T2DM, } \\
\text { hyperlipidemia, HT }\end{array}$ \\
\hline $\begin{array}{l}\text { Fung et al. } \\
2009 \text { [38] }\end{array}$ & U.K. & Women & $38-63$ & 20 & 74,886 & $\begin{array}{l}\text { aMED [45]: }(+) \text { vegetables } \\
\text { (excluding potatoes), fruits, } \\
\text { nuts, whole grain, legumes, } \\
\text { fish, MUFA/SFA; }(+\mathrm{m}) \mathrm{OH} \text {; } \\
(-) \text { meat }\end{array}$ & Risk of stroke & $\begin{array}{l}\mathrm{Q} 5 \downarrow, \text { risk CHD RR } 0.71 \text { (95\% CI 0.62-0.82), } \\
p=0.0001 \text { for CHD; } \\
\downarrow \text { risk stroke RR } 0.87 \text { (95\% CI 0.73-1.02), } \\
p=0.03 \text {; } \\
\text { Q5, } \downarrow \text { CVD mortality } q \text { RR } 0.61 \\
(95 \% \text { CI } 0.49-0.76), p=0.0001\end{array}$ & $\begin{array}{l}\text { Age, smoking, BMI, } \\
\text { menopausal status, } \\
\text { postmenopausal hormone } \\
\text { use, TEI, multivitamin } \\
\text { intake, OH intake, family } \\
\text { history, PA, aspirin }\end{array}$ \\
\hline $\begin{array}{c}\text { Gardener et al. } \\
2011 \text { [32] }\end{array}$ & EEUU (NY) & $\begin{array}{l}\text { Men and } \\
\text { women }\end{array}$ & $69 \pm 10$ & 9 & 2568 & $\begin{array}{l}\text { Own MD Score [45]: dairy, } \\
\text { meat, fruit, vegetables } \\
\text { (excluding potatoes), legumes, } \\
\text { cereals (refined and whole), fish }\end{array}$ & CHD events & $\begin{array}{l}\text { Q5 score } \\
\text { (1) HR } 0.75(95 \% \text { CI } 0.56-0.99) p=0.04 \text {; } \\
\text { (2) HR } 0.80(0.60-1.06) p=0.10 \text {. } \\
\text { With 1P increase score } \\
\text { (1) HR } 0.94(0.89-1.00) \text {, } \\
\text { (2) HR } 0.95(0.90-1.01)\end{array}$ & $\begin{array}{l}\text { (1) Age, sex, ethnicity, } \\
\text { education, PA, TEI, } \\
\text { smoking; (2) model } 1 \\
\text { and T2DM, HT, } \\
\text { hypercholesterolemia, } \\
\text { history self-reported } \\
\text { cardiac disease }\end{array}$ \\
\hline
\end{tabular}


Table 1. Cont

\begin{tabular}{|c|c|c|c|c|c|c|c|c|c|}
\hline $\begin{array}{l}\text { Author/Year } \\
\text { of Publication }\end{array}$ & Country & Gender & $\begin{array}{c}\text { Age } \\
\text { (Years) }\end{array}$ & $\begin{array}{c}\text { Follow-Up } \\
\text { (Years) }\end{array}$ & $N$ & Components in the MD Index & $\begin{array}{c}\text { Main } \\
\text { Outcome } \\
\end{array}$ & Results & Confounders \\
\hline $\begin{array}{l}\text { Martínez-Glez et al. } \\
\qquad 2011 \text { [75] }\end{array}$ & Spain & $\begin{array}{l}\text { Men and } \\
\text { women }\end{array}$ & Mean 38 & 4.9 & 13,609 & $\begin{array}{l}\text { 9-Point score [45]: }(+) \text { MUFA/SFA, } \\
\text { legumes, cereals, fruits, nuts, } \\
\text { vegetables, fish; }(+\mathrm{m}) \mathrm{OH} ; \\
(-) \text { total dairy products, meat }\end{array}$ & $\begin{array}{l}\text { CHD } \\
\text { events }\end{array}$ & $\begin{array}{l}\uparrow \mathrm{MD}, \downarrow \mathrm{CVD} \text { risk HR } 0.41 \text { (95\% CI 0.18-0.95); } \\
\text { with } 2 \text { P increase score, } \\
\text { total CVD HR } 0.80 \text { (95\% CI 0.62-1.02), } \\
\text { CHD HR } 0.74 \text { (95\% CI } 0.55-0.99)\end{array}$ & $\begin{array}{l}\text { Age, sex, TEI, family } \\
\text { history CHD, smoking, } \\
\text { PA, baseline BMI, HT } \\
\text { or use of medication, } \\
\text { aspirin, baseline T2DM, } \\
\text { baseline dyslipidaemia }\end{array}$ \\
\hline $\begin{array}{c}\text { Menotti et al. } \\
2012 \text { [76] }\end{array}$ & Italy & $\begin{array}{l}\text { Men and } \\
\text { women }\end{array}$ & $45-64$ & 5 & 1139 & $\begin{array}{l}\text { MAI [77]: }(+) \text { cereals, legumes, } \\
\text { potatoes, vegetables, fresh dry fruit, } \\
\text { fish, wine, virgin olive oil; }(-) \text { milk, } \\
\text { cheese, meat, eggs, animal fats and } \\
\text { margarines, sweet beverages, sugar }\end{array}$ & $\begin{array}{l}\text { CHD } \\
\text { events }\end{array}$ & $\begin{array}{l}20 \text {-Year follow-up lnMAI } \\
\text { HR } 0.74(95 \% \text { CI } 0.56-0.98) p=0.0338 \text {; } \\
\text { 40-year follow-up lnMAI } \\
\text { HR } 0.79(95 \% \text { CI } 0.65-0.96) p=0.0168\end{array}$ & $\begin{array}{l}\text { Age, cigarette smoking, } \\
\text { systolic blood pressure, } \\
\text { serum cholesterol, PA, BMI }\end{array}$ \\
\hline $\begin{array}{l}\text { Guallar-Castrillón et al. } \\
\qquad 2012 \text { [78] }\end{array}$ & Spain & $\begin{array}{l}\text { Men and } \\
\text { women }\end{array}$ & $29-69$ & $\begin{array}{c}8-12 ; \\
\text { median } 11\end{array}$ & 40,757 & Own score & $\begin{array}{l}\text { CHD } \\
\text { events }\end{array}$ & $\begin{array}{l}\text { MD score HR } \\
\text { Q2 } 0.77 \text { (95\% CI } 0.61-0.98) \text {, } \\
\text { Q3 } 0.64 \text { (95\% CI } 0.50-0.83) \text {, } \\
\text { Q4 } 0.56 \text { (95\% CI } 0.43-0.73) \text {, } \\
\text { Q5 } 0.73 \text { (95\% CI } 0.57-0.94)\end{array}$ & $\begin{array}{l}\text { Age, sex, BMI, WC, } \\
\text { educational level, smoking, } \\
\text { PA at work, home and } \\
\text { leisure time, T2DM, HT, } \\
\text { hypercholesterolemia, } \\
\text { cancer, oral contraceptives, } \\
\text { menopausal status, } \\
\text { hormone replacement } \\
\text { therapy, TEI }\end{array}$ \\
\hline
\end{tabular}


Table 1. Cont.

\begin{tabular}{|c|c|c|c|c|c|c|c|c|c|}
\hline $\begin{array}{l}\text { Author/Year } \\
\text { of Publication }\end{array}$ & Country & Gender & $\begin{array}{c}\text { Age } \\
\text { (Years) }\end{array}$ & $\begin{array}{c}\text { Follow-Up } \\
\text { (Years) }\end{array}$ & $N$ & Components in the MD Index & $\begin{array}{c}\text { Main } \\
\text { Outcome }\end{array}$ & Results & Confounders \\
\hline $\begin{array}{l}\text { Estruch et al. } \\
2013[46]\end{array}$ & Spain & $\begin{array}{l}\text { Men and } \\
\text { women }\end{array}$ & $55-80$ & 4.8 & 7447 & PREDIMED [51] & CHD events & $\begin{array}{l}\text { MD VOO HR } 0.70 \text { (95\% CI 0.54-0.92); } \\
\text { MD nuts HR } 0.72(95 \% \text { CI } 0.54-0.96)\end{array}$ & $\begin{array}{l}\text { Sex, age, BMI, } \\
\text { cardiovascular-risk-factor status, } \\
\text { baseline adherence MedDiet }\end{array}$ \\
\hline
\end{tabular}

MD, Mediterranean Diet; $\hat{0}$ men; $\rho$ women; MUFA/SFA, ratio of monounsaturated to saturated fat; Meat, meat and meat products; Dairy, milk and dairy products; $(+)$, positive components; $(-)$, negative components; (+m), components positive in moderation; (d) daily; (w) weekly; (m) monthly; adh, adherence; HR, hazard ratio; OH, alcohol consumption; PA, physical activity; TEI, total energy intake; WC, waist circumference; hc, hip circumference; HT, hypertension; CI, confidence interval; OR: odds ratio; BMI, body mass index; T2DM, type 2 diabetes mellitus; ARR, absolute risk reduction; RRR, relative risk reduction; Q, quartile; P, points; $p$ for trend; MetS, metabolic syndrome; MDS, Mediterranean diet score; MED, Mediterranean score; MSDPS, Mediterranean style-dietary pattern score; HC, high-carbohydrate diet group; HF, high-fat diet group; T, tertile; mMDS, modified-Mediterranean Diet Score; rMED, Mediterranean Diet Score; LTPA, leisure-time physical activity; OW, overweight; NW, normal weight; S, significant; NS, not significant; WHtR, waist-to-height ratio; CHD, coronary heart disease; ACS, acute coronary syndrome; MAI, Mediterranean Adequacy Index; DM, diabetes mellitus; VOO, virgin olive oil; CVD, cardiovascular disease; 10 European countries (Denmark, France, Germany, Greece, Italy, the Netherlands, Norway, Spain, Sweden, the United Kingdom). 


\subsection{Association between the MedDiet and Cardiodiabesity}

Out of the 37 studies, 33 reported a significant association between adherence to MedDiet and reduced incidence and prevalence of cardiodiabesity, but in four there was no association between MedDiet, T2DM, obesity, MetS and CVD [33,37,54,65]. Figure 2 describes the overall diet-related cardiodiabesity parameters and cardiovascular risk factors used in the different studies reviewed.

Figure 2. Diet-related Cardiodiabesity diagnostic parameters. Abbreviations are as follows: METS, metabolic syndrome; T2DM, type 2 diabetes mellitus; CVD, cardiovascular disease; WC, waist circumference; BMI, body mass index.
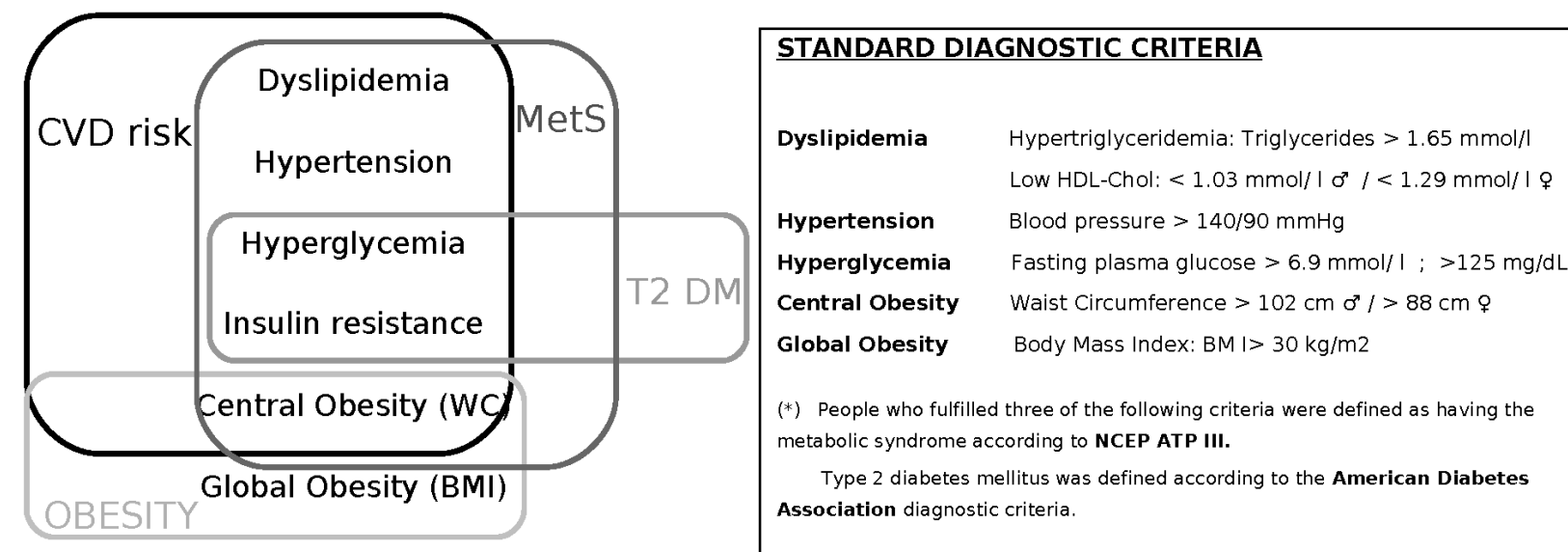

Regarding the association between adherence to the MedDiet pattern and T2DM, Abiemo et al. [33] reported that a greater consistency with a MedDiet style was cross-sectionally associated with lower serum insulin concentrations among non-diabetics and with lower blood glucose before adjustment for obesity but not with a lower incidence of DM. However, three out of the four studies selected found that a higher MedDiet score was predictive of a lower incidence of T2DM [47,49,50]. The greatest relative reduction in T2DM risk (52\%) was observed in the PREDIMED nutritional intervention trial in subjects at high CVD risk who showed high adherence to the traditional MedDiet [50]. The incidence of T2DM was reduced by 35\% in the cohort study of Martínez-González et al. [49] in association with a two-point increase in the Trichopoulou score, adjusting for confounders. Panagiotakos et al. [47] described that a 10-unit increase in the diet score was associated with $21 \%$ lower odds of T2DM, while the combination of greater adherence to the MedDiet and light physical activity reduced the risk of diabetes by $35 \%$ after adjustment for various factors.

The impact of the MedDiet pattern on the prevalence of the MetS was evaluated in five cross-sectional studies selected in this review $[43,52,54,58,61]$. In all these studies, the MetS criteria were defined according to the National Cholesterol Education Program (NCEP) Adult Treatment Panel (ATP) III [79], which includes hypertriglyceridemia, low HDL-cholesterol, hypertension, hyperglycemia and central obesity. People who fulfilled three or more of these conditions were defined as having MetS. Alvarez-León et al. [54] concluded that MedDiet adherence was not related to MetS prevalence even though some components of the MedDiet showed a protective effect on the MetS and its components. The ATTICA study [52] determined that the odds ratio of having MetS decreased when the participants consumed a MedDiet and reported little-to-moderate physical activity. Other 
cross-sectional studies reported significant inverse associations between high adherence to the MetDiet and the prevalence of MetS criteria in elderly men with high CVD risk [58], in adolescents [43] and in overweight/obese subjects [61], respectively. Moreover, the three cohort studies selected, with a follow-up of 6 to 7 years, found the lowest incidence of MetS in subjects with the highest adherence to the MedDiet [34,56,62]. In addition, the overall prevalence of MetS at the first year assessment of the PREDIMED cohort showed a higher reduction in participants who consumed a MedDiet supplemented with mixed nuts compared with patients given advice to follow a low-fat diet [57]. It should be noted that in some studies individual components of the MetS, especially hyperglycemia, were not always significantly affected $[56,57,62]$.

Most of the studies selected showed that the prevalence of overweight, obesity and central obesity was inversely associated with the MedDiet score. Classified according to study type, three cohort studies $[67,69,70]$ reported that adherence to a MedDiet pattern was significantly associated with a reduced weight gain, and that the risk to develop overweight and obesity was also less likely. Specifically, Mendez et al. [67] reported a decrease in obesity with MedDiet adherence, albeit only being significant among the overweight population. All the interventional dietary studies $[35,40,73]$ found that adherence to a MedDiet significantly decreased weight/BMI and, specifically, abdominal obesity. Regarding the cross-sectional studies, the EPIC-PANACEA study by Romaguera et al. [37] found that higher adherence to the MedDiet was significantly associated with lower abdominal adiposity for a given BMI, measured by waist circumference, while the MedDiet was not significantly associated with general obesity (BMI). Tripocholou et al. [65] did not find any association between MedDiet adherence and weight either. In contrast, five of the seven cross-sectional studies $[36,41,64,66,68]$ found that greater adherence to a MedDiet had a significantly negative association with overweight/obesity. The strongest association was reported in the ATTICA study by Panagiatakos et al. [66] who found that individuals with high MedDiet adherence were $51 \%$ less likely to have both central adiposity and obesity, after adjustment for potential confounders. Note that in the CYKIDS study by Lazarou et al. [41] although adherence to the MedDiet was inversely associated with childhood obesity, when physical activity was taken into account, this relationship became less significant. Furthermore, children with higher adherence to a MedDiet reported to practice higher physical activity levels [42].

All the studies on the primary prevention of CVD showed a statistically significant association between the MedDiet pattern and the incidence of CVD [32,38,39,45,46,71,74-76,78]. In the PREDIMED trial, the largest randomized trial aimed to assess the effect of a dietary intervention using MedDiet on CVD outcomes; Estruch et al. [46] found that among persons at high cardiovascular risk, a MedDiet supplemented with extra-virgin olive oil or mixed nuts reduced the incidence of major cardiovascular events by $30 \%$. Regarding cohort studies, adherence to the MedDiet was associated with a lower risk of coronary heart disease (CHD) in the large Spanish cohort of the EPIC study [71,75,78]. This MedDiet association has been assessed by either by using a priori [71,75] or a posteriori scales [78] during a median follow-up period of 10 years. The longest follow-up period was conducted in the Italian cohort study by Menotti et al. [76] in which individuals with a higher adherence to the MedDiet showed a protective effect against the occurrence of fatal CHD events at 20 and 40 years (CHD mortality reduction of $26 \%$ and $22 \%$, respectively). Additionally, Tripochoulou et al. [45] (from the Greek cohort of the EPIC study) reported that the MedDiet was 
associated with a significant reduction in mortality due to CHD. Moreover, an inverse association with greater adherence to a MedDiet was observed for all-cause mortality and cause-specific death such as CHD and CVD [39], lower risk of stroke [38] and other vascular events, such as myocardial infarction and vascular death [32].

\section{Discussion}

This review is focused on the potential beneficial role of a MedDiet pattern for overall primary cardiodiabesity prevention taking into account the most solid, epidemiological and updated scientific evidence available. Several reviews have previously reported the beneficial effect of the MedDiet on obesity [30], T2DM, MetS [80] and CVD [81] risk factors. In this review, cardiodiabesity is considered as a term that embodies the overall diet-related diseases, which are the first causes of death worldwide, as well as others that affect and worsen health conditions.

Of the 37 studies reviewed in this report, 33 provided strong evidence on the association between adherence to a MedDiet and CDV, T2DM, MetS and obesity. Moreover, results from the large scale randomized intervention study, the PREDIMED trial, have pointed out that after 5 years of intervention among high-risk persons who were initially free of CVD, a MedDiet (with no caloric restriction) supplemented with one daily serving of mixed nuts or up to $50 \mathrm{~mL}$ of extra-virgin olive oil reduced the incidence of three major cardiovascular events (cardiovascular death, myocardial infarction and stroke) [46] by 30\%, suggesting a higher protective effect against cardiovascular risk factors with a MedDiet compared to a low-fat diet, with the added strength of higher compliance among the followers of the MedDiet [46,51]. This protective effect of the MedDiet observed appears early after starting the dietary change and is similar to the effects of statin and antihypertensive drug treatments but without side effects. In addition, the Portfolio diet described by Jenkins and colleagues, which comprised four key components as foods rich in soluble fiber, soy protein, plant sterols and almonds, reported a $29 \%$ reduction in LDL-cholesterol comparable to that observed with a small dose of a statin [82].

To increase the quality and level of scientific evidence, in this review we included prospective observational cohorts and clinical trials. Case-control studies were excluded because of the related bias as mentioned. Nevertheless, the results among the studies reviewed were not completely consistent. Romaguera et al. [37] and Trichopoulou et al. [65] found no significant relationship between adherence to a MedDiet and BMI, but they did find a relationship with a lower waist circumference for a given BMI in both genders. Abiemo et al. [33] reported that higher adherence to MedDiet was associated with lower insulin concentrations even though it did not decrease the incidence of T2DM, and the MeDiet also showed protective effects on some MetS components although the Alvarez et al. [54] study did not fulfill the conditions that — according to NCEP-ATPIII criteria - more than three components have to be present to diagnose MetS. Thus some studies reported no clear association between adherence to a traditional MedDiet and main outcomes although they did find positive associations with intermediate risks factors $[33,37,54,65]$. The variability of the associations observed and the strength between adherence to a MedDiet and cardiodiabesity could also be linked to several aspects and limitations inherent to differences between studies and samples. Similarly, other potential limitations were adjustments for different confounders and indexes used as described in the 
table. Moreover, specifically designed studies should be carried out to assess these outcomes simultaneously in order to determine the synergy of interventions in several health parameters involved in cardiodiabesity.

According to the American Public Health Association (APHA), in the USA obesity has about 152 billion dollars/year and 73 billion dollars/year in direct and indirect (lost productivity, absenteeism, etc.) costs, respectively [83]. In the current economic context, variables related to health management and the cost-benefit ratio are particularly relevant to health policies and clinical protocols, and, thus, to action guidelines. Current recommendations highlight the importance of dietary counseling in clinical practice. Even though the MedDiet is a diet with a high fat intake, and fat sources are derived primarily from monounsaturated oils, in their last guideline on T2DM, pre-diabetes, and CVD [6], the European Society of Cardiology together with the European Association for the Study of Diabetes group (ESC/EASD) stated, that the MedDiet is an acceptable alternative to the traditionally proposed DASH diets, which are mostly prescribed in clinical practice today. Besides, ESC/EASD support the consideration that T2DM treatment and prevention should be firmly based on complex and non-pharmacological therapies [84]. The ADA 2013 nutrition therapy recommendations provide a summary of strong scientific evidence supporting the effectiveness of nutrition therapy across the continuum of diabetes management [84], which has reported that non-drug treatment is as effective as hypoglycemic drug-treatment, involving a mean reduction of $1.0 \%-1.5 \%$ in HbA1c [84]. The role of nutrition in the prevention of CVD has been extensively reviewed [85-87] and strong evidence of the influence of dietary factors in atherogenesis both directly and through effects on traditional risk factors, such as lipid profile, blood pressure or plasma glucose concentration has been reported [88]. Thus, management of obesity and prevention of CVD and T2DM should be aimed at reducing the overall risk of cardiodiabesity in a holistic manner.

Despite the beneficial effects of the MedDiet, there are discrepancies among nutrition experts because of the high-fat content of this diet (up to $>40 \%$ of total energy intake), which is in conflict with the usual recommendation to follow a low-fat diet in order to avoid overweight/obesity and to prevent CHD [89-91]. A recent meta-analysis [92] argued that current evidence does not clearly support cardiovascular guidelines that encourage high consumption of polyunsaturated fatty acids and low consumption of total saturated fats. However, it should be remarked that the PREDIMED trial demonstrated with the highest level of scientific evidence that the MedDiet is a useful tool to prevent CVD end-points in high-risk subjects [46]. On the other hand, the higher palatability, acceptance and compliance with MedDiets in comparison with low-fat diets should also be taken into account [93].

This review shows that most of the diet-related risk factors of cardiodiabesity may be preventable through healthy lifestyles and specifically with a healthy dietary pattern. Note that all the studies included took into account the physical activity of the participants but no study undertook active intervention controlling of this variable. Thus, all evidence on the reported beneficial effect was mainly due to the overall composition of the dietary pattern, without other lifestyle changes. Preventive medicine should be part of current and future public health strategies in the global context of high rates of chronic diseases around the world. Nowadays, important cultural and economic changes are affecting the population lifestyle, and consequently its health. In recent decades, there has been a strong social transformation that has led to poorer food habits, a decline in traditional customs and a change in the MedDiet pattern [48,91,94]. Promotion of a healthy lifestyle can be used for the 
prevention or treatment of several factors contributing to cardiodiabesity and lead to the avoidance of progression and the need for pharmacological interventions. The Spanish Society of Family and Community Medicine (SEMFYC) agrees that there is sufficient evidence on the benefits of the dietary advice carried out by trained personnel on the prevention of several chronic diseases, but, on the other hand, there is little evidence on the effectiveness of dietary advice in primary care, probably due to inadequate dietary assessment and other factors. Thus, the dietary advice targeted at apparently healthy or low-risk asymptomatic individuals should be simple and easy to comply with [95]. The new MedDiet pyramid [18] is the result of an international consensus based on the latest scientific evidence on nutrition and health, and provides key elements for the quantitative and qualitative selection of foods. This new MedDiet pyramid is meant for use and promotion without any restrictions, and the nutritional recommendations based on a MedDiet pattern may lead to the prevention of chronic diseases, especially if dietary recommendations take into account individual preferences, thereby ensuring long-time adherence. In this sense, the MedDiet could be translated into daily clinical primary care practice in the setting of public health promotion programs. In order to facilitate this step and achieve greater compliance with dietary advice, the development of the following points should be taken into account:

(i) To develop simple, objective and useful tools to determine the degree of patient adherence to the MedDiet pattern for its application in the clinical setting and general practice.

(ii) To establish consensual recommendations about the MedDiet pattern and psychological approaches to eating habits and lifestyle improvements. Moreover, it is important to foster knowledge of and accessibility to this dietary pattern to health professionals, particularly in primary care where preventive medicine is of greatest, albeit not exclusive, significance. Multidisciplinary approaches and strategies are needed to increase compliance to treatments and even dietary and lifestyle interventions [96].

\section{Conclusions}

Most of the reviewed studies provided strong evidence on the association between adherence to a MedDiet and CDV, T2DM, MetS and obesity, remarking the relationship between all these interconnected illnesses and supporting the term cardiodiabesity. The results of the current review of epidemiological and clinical trial studies support the role of the MedDiet in the prevention of cardiodiabesity. The prevention of cardiodiabesity by good adherence to the MedDiet is supported by the latest most solid scientific evidence, and further by its low environmental footprints and economic accessibility in Mediterranean countries. Furthermore, the high palatability of the MedDiet makes this dietary pattern very useful for preventive strategies applied to the general population in primary care medicine for optimal collaborative management of these patients.

\section{Author Contributions}

Elena, G.-F. and Anna, B.-F. designed the review. All authors participated in the process, although Elena, G.-F. conducted the PubMed search and made the table. Laura, R.-C. wrote the manuscript and 
thereafter all the authors, Elena, G.-F.; Anna, B.-F.; Nanna, R. and Ramón, E. contributed to the writing of the text and drafting of the figures.

\section{Conflicts of Interest}

The authors declare no conflict of interest related to the current article.

\section{References}

1. Shape up America! Available online: http://www.shapeup.org/ (accessed on 19 June 2014).

2. Robertson, D. Cardiodiabetes-Is a joint approach the way forward? Br. J. Cardiol. 2008, 15, S8-S10.

3. World Health Organization Media Centre (WHO). Diabetes. Available online: http://www. who.int/mediacentre/factsheets/fs312/es/ (accessed on 12 January 2014).

4. World Health Organization Media Centre (WHO). 10 Facts on Obesity. Available online: http://www.who.int/features/factfiles/obesity/es/ (accessed on 12 January 2014).

5. World Health Organization Media Centre (WHO). Cardiovascular Diseases. Available online: http://www.euro.who.int/en/health-topics/noncommunicable-diseases/cardiovascular-diseases/ cardiovascular-diseases2 (accessed on 12 January 2014).

6. Rydén, L.; Grant, P.J.; Anker, S.D.; Berne, C.; Cosentino, F.; Danchin, N.; Deaton, C.; Escaned, J.; Hammes, H.P.; Huikuri, H.; et al. ESC Guidelines on diabetes, pre-diabetes, and cardiovascular diseases developed in collaboration with the EASD: The Task Force on diabetes, pre-diabetes, and cardiovascular diseases of the European Society of Cardiology (ESC) and developed in collaboration with the European Association for the Study of Diabetes (EASD). Eur. Heart J. 2013, 34, 3035-3087.

7. National Institute for Health and Clinical Excellence (NICE). Clinical Guideline 67. Lipid Modification. Cardiovascular Risk Assessment and the Modification of Blood Lipids for the Primary and Secondary Prevention of Cardiovascular Disease, May 2008. Available online: http://www.nice.org.uk/guidance/cg67/chapter/guidance (accessed on 25 January 2014).

8. National Institute for Health and Clinical Excellence (NICE). Clinical Guideline 66. Type 2 Diabetes, May 2008. Available online: http://www.nice.org.uk/guidance/cg87 (accessed on 25 January 2014)

9. Grundy, S.M. Hypertriglyceridemia, atherogenic dyslipidemia and the metabolic syndrome. Am. J. Cardiol. 1998, 81, 18B-25B.

10. Grundy, S.M. Hypertriglyceridemia, insulin resistance and the metabolic syndrome. Am. J. Cardiol. 1999, 83, 25F-29F.

11. Hajer, G.H.; van Haeften, T.W.; Visseren, F. Adipose tissue dysfunction in obesity, diabetes, and vascular diseases. Eur. Heart J. 2008, 29, 2959-2971.

12. Martinez-Gonzalez, M.A.; Bes-Rastrollo, M.; Serra-Majem, L.; Lairon, D.; Estruch, R.; Trichopoulou, A. Mediterranean food pattern and the primary prevention of chronic disease: Recent developments. Nutr. Rev. 2009, 67, S111-S116.

13. Serra-Majem, L.; Roman, B.; Estruch, R. Scientific evidence of interventions using the Mediterranean diet: A systematic review. Nutr. Rev. 2006, 64, S27-S47. 
14. Sofi, F.; Cesari, F.; Abbate, R.; Gensini, G.F.; Casini, A. Adherence to Mediterranean diet and health status: Meta-Analysis. BMJ 2008, 337, a1344, doi:10.1136/bmj.a1344.

15. Sofi, F.; Abbate, R.; Gensini, G.F.; Casini, A. Accruing evidence on benefits of adherence to the Mediterranean diet on health: An updated systematic review and meta-analysis. Am. J. Clin. Nutr. 2010, 92, 1189-1196.

16. Sofi, F.; Macchi, C.; Abbate, R.; Gensini, G.F.; Casini, A. Mediterranean diet and health status: An updated meta-analysis and a proposal for a literature-based adherence score. Public Health Nutr. 2013, 29, 1-14.

17. Serra-Majem, L.; Bes-Rastrollo, M.; Román, B.; Pfrimer, K.; Sánchez-Villegas, A.; Martínez-González, M.A. Dietary patterns and nutritional adequacy in a Mediterranean country. Br. J. Nutr. 2009, 101, S21-S28.

18. Bach-Faig, A.; Berry, E.M.; Lairon, D.; Reguant, J.; Trichopoulou, A.; Dernini, S.; Medina, F.X.; Battino, M.; Belahsen, R.; Miranda, G.; et al. Mediterranean diet pyramid today. Science and cultural updates. Public Health Nutr. 2011, 14, 2274-2284.

19. Consenso FESNAD-SEEDO. Recomendaciones nutricionales basadas en la evidencia para la prevención y el tratamiento del sobrepeso y la obesidad en adultos. Rev. Esp. Obes. 2011, 10, 1-78.

20. Kant, A.K. Dietary patterns and health outcomes. J. Am. Diet. Assoc. 2004, 104, 615-635.

21. Bach-Faig, A.; Serra-Majem, L.; Carrasco, J.L.; Roman, B.; Ngo, J.; Bertomeu, I.; Obrador, B. The use of indexes evaluating the adherence to the Mediterranean diet in epidemiological studies: A review. Public Health Nutr. 2006, 9, 132-146.

22. UNESCO. Representative List of the Intangible Cultural Heritage of Humanity-2010. Available online: http://www.unesco.org/culture/ich/en/RL/00394 (accessed on 28 January 2014).

23. Sáez-Almendros, S.; Obrador, B.; Bach-Faig, A.; Serra-Majem, L. Environmental footprints of Mediterranean versus Western dietary patterns: Beyond the health benefits of the Mediterranean diet. Environ. Health 2013, 12, 118, doi:10.1186/1476-069X-12-118.

24. Saulle, R.; Semyonov, L.; la Torre, G. Cost and cost-effectiveness of the Mediterranean diet: Results of a systematic review. Nutrients 2013, 5, 4566-4586.

25. Da Silva, R.; Bach-Faig, A.; Raido Quintana, B.; Buckland, G.; vaz de Almeida, M.D.; Serra-Majem, L. Worldwide variation of adherence to the Mediterranean diet, in 1961-1965 and 2000-2003. Public Health Nutr. 2009, 12, 1676-1684.

26. Serra-Majem, L.; Helsing, E. Changing patterns of fat intake in Mediterranean countries. Eur. J. Clin. Nutr. 1993, 47, S13-S20.

27. Ferro-Luzzi, A.; Sette, S. The Mediterranean diet: An attempt to define its present and past composition. Eur. J. Clin. Nutr. 1989, 43, 13-29.

28. Panagiotakos, D.B.; Pitsavos, C.; Polychronopoulos, E.; Chrysohoou, C.; Zampelas, A.; Trichopoulou, A. Can a Mediterranean diet moderate the development and clinical progression of coronary heart disease? A systematic review. Med. Sci. Monit. 2004, 10, RA193-RA198.

29. De Lorgeril, M.; Salen, P. The Mediterranean-style diet for the prevention of cardiovascular diseases. Public Health Nutr. 2006, 9, 118-123.

30. Buckland, G.; Bach, A.; Serra-Majem, L. Obesity and the Mediterranean diet: A systematic review of observational and intervention studies. Obes. Rev. 2008, 9, 582-593. 
31. Nordmann, A.J.; Suter-Zimmermann, K.; Bucher, H.C.; Shai, I.; Tuttle, K.H.; Estruch, R.; Briel, M. Meta-Analysis comparing Mediterranean to low-fat diets for modification of cardiovascular risk factors. Am. J. Med. 2011, 124, 841-851.

32. Gardener, H.; Wright, C.B.; Gu, Y.; Demmer, R.T.; Boden-Albala, B.; Elkind, M.S.; Sacco, R.L.; Scarmeas, N. Mediterranean-style diet and risk of ischemic stroke, myocardial infarction, and vascular death: The Northern Manhattan Study. Am. J. Clin. Nutr. 2011, 94, 1458-1464.

33. Abiemo, E.E.; Alonso, A.; Nettleton, J.A.; Steffen, L.M.; Bertoni, A.G.; Jain, A.; Lutsey, P.L. Relationships of the Mediterranean dietary pattern with insulin resistance and diabetes incidence in the Multi-Ethnic Study of Atherosclerosis (MESA). Br. J. Nutr. 2013, 109, 1490-1497.

34. Rumawas, M.E.; Meigs, J.B.; Dwyer, J.T.; McKeown, N.M.; Jacques, P.F. Mediterranean-style dietary pattern, reduced risk of metabolic syndrome traits, and incidence in the Framingham Offspring Cohort. Am. J. Clin. Nutr. 2009, 90, 1608-1614.

35. Goulet, J.; Lamarche, B.; Nadeau, G.; Lemieux, S. Effect of a nutritional intervention promoting the Mediterranean food pattern on plasma lipids, lipoproteins and body weight in healthy French-Canadian women. Atherosclerosis 2003, 170, 115-124.

36. Shubair, M.M.; McColl, R.S.; Hanning, R.M. Mediterranean dietary components and body mass index in adults: The peel nutrition and heart health survey. Chronic Dis. Can. 2005, 26, 43-51.

37. Romaguera, D.; Norat, T.; Mouw, T.; May, A.M.; Bamia, C.; Slimani, N.; Travier, N.; Besson, H.; Luan, J.; Wareham, N. Adherence to the Mediterranean diet is associated with lower abdominal adiposity in European men and women. J. Nutr. 2009, 139, 1728-1737.

38. Fung, T.T.; Rexrode, K.M.; Mantzoros, C.S.; Manson, J.E.; Willett, W.C.; Hu, F.B. Mediterranean diet and incidence of and mortality from coronary heart disease and stroke in women. Circulation 2009, 119, 1093-1100.

39. Knoops, K.T.; de Groot, L.C.; Kromhout, D.; Perrin, A.E.; Moreiras-Varela, O.; Menotti, A.; van Staveren, W.A. Mediterranean diet, lifestyle factors, and 10-year mortality in elderly European men and women: The HALE project. JAMA 2004, 292, 1433-1439.

40. Andreoli, A.; Lauro, S.; di Daniele, N.; Sorge, R.; Celi, M.; Volpe, S.L. Effect of a moderately hypoenergetic Mediterranean diet and exercise program on body cell mass and cardiovascular risk factors in obese women. Eur. J. Clin. Nutr. 2008, 62, 892-897.

41. Lazarou, C.; Panagiotakos, D.B.; Matalas, A.L. Physical activity mediates the protective effect of the Mediterranean diet on children's obesity status: The CYKIDS study. Nutrition 2010, 26, 61-67.

42. Farajian, P.; Risvas, G.; Karasouli, K.; Pounis, G.D.; Kastorini, C.M.; Panagiotakos, D.B.; Zampelas, A. Very high childhood obesity prevalence and low adherence rates to the Mediterranean diet in Greek children: The GRECO study. Atherosclerosis 2011, 217, 525-530.

43. Bibiloni, M.; Martínez, E.; Llull, R.; Maffiotte, E.; Riesco, M.; Llompart, I.; Pons, A.; Tur, J.A. Metabolic syndrome in adolescents in the Balearic Islands, a Mediterranean region. Nutr. Metab. Cardiovasc. Dis. 2011, 21, 446-454.

44. Trichopoulou, A.; Kouris-Blazos, A.; Wahlqvist, M.L.; Gnardellis, C.; Lagiou, P.; Polychronopoulos, E.; Vassilakou, T.; Lipworth, L.; Trichopoulos, D. Diet and overall survival in elderly people. Br. Med. J. 1995, 311, 1457-1460. 
45. Trichopoulou, A.; Costacou, T.; Bamia, C.; Trichopoulos, D. Adherence to a Mediterranean diet and survival in a Greek population. N. Engl. J. Med. 2003, 348, 2599-2608.

46. Estruch, R.; Ros, E.; Salas-Salvadó, J.; Covas, M.I.; Corella, D.; Arós, F.; Gómez-Gracia, E.; Ruiz-Gutiérrez, V.; Fiol, M.; Lapetra, J.; et al. Primary prevention of cardiovascular disease with a Mediterranean diet. N. Engl. J. Med. 2013, 368, 1279-1290.

47. Panagiotakos, D.B.; Pitsavos, C.; Chrysohoou, C.; Stefanadis, C. The epidemiology of Type 2 diabetes mellitus in Greek adults: The ATTICA study. Diabet. Med. 2005, 22, 1581-1588.

48. Willett, W.C.; Sacks, F.; Trichopoulou, A.; Drescher, G.; Ferro-Luzzi, A.; Helsing, E.; Trichopoulos, D. Mediterranean diet pyramid: A cultural model for healthy eating. Am. J. Clin. Nutr. 1995, 61, 1402S-1406S.

49. Martínez-González, M.A.; de la Fuente-Arrillaga, C.; Nunez-Cordoba, J.M.; Basterra-Gortari, F.J.; Beunza, J.J.; Vazquez, Z.; Benito, S.; Tortosa, A.; Bes-Rastrollo, M. Adherence to Mediterranean diet and risk of developing diabetes: Prospective cohort study. BMJ 2008, 336, 1348-1351.

50. Salas-Salvadó, J.; Bulló, M.; Babio, N.; Martínez-González, M.Á.; Ibarrola-Jurado, N.; Basora, J.; Estruch, R.; Covas, M.I.; Corella, D.; Arós, F.; et al. PREDIMED Study Investigators. Reduction in the incidence of type 2 diabetes with the Mediterranean diet: Results of the PREDIMED-Reus nutrition intervention randomized trial. Diabetes Care 2011, 34, 14-19.

51. Estruch, R.; Martínez-González, M.A.; Corella, D.; Salas-Salvadó, J.; Ruiz-Gutiérrez, V.; Covas, M.I.; Fiol, M.; Gómez-Gracia, E.; López-Sabater, M.C.; Vinyoles, E.; et al. Effects of a Mediterranean-style diet on cardiovascular risk factors: A randomized trial. Ann. Intern. Med. 2006, 145, 1-11.

52. Panagiotakos, D.B.; Pitsavos, C.; Chrysohoou, C.; Skoumas, J.; Tousoulis, D.; Toutouza, M.; Toutouzas, P.; Stefanadis, C. Impact of lifestyle habits on the prevalence of the metabolic syndrome among Greek adults from the ATTICA study. Am. Heart J. 2004, 147, 106-112.

53. Trichopoulou, A. From research to education: The Greek experience. Nutrition 2000, 16, 528-531.

54. Alvarez León, E.; Henríquez, P.; Serra-Majem, L. Mediterranean diet and metabolic syndrome: A cross-sectional study in the Canary Islands. Public Health Nutr. 2006, 9, 1089-1098.

55. Henríquez, P.; Doreste, J.; Díaz-Cremades, J.; López-Blanco, F.; Alvarez-León, E.; Serra-Majem, L. Folate status of adults living in the Canary Islands (Spain). Int. J. Vit. Nutr. Res. 2004, 74, 187-192.

56. Tortosa, A.; Bes-Rastrollo, M.; Sanchez-Villegas, A.; Basterra-Gortari, F.J.; Nuñez-Cordoba, J.M.; Martinez-Gonzalez, M.A. Mediterranean diet inversely associated with the incidence of metabolic syndrome: The SUN prospective cohort. Diabetes Care 2007, 30, 2957-2959.

57. Salas-Salvadó, J.; Fernández-Ballart, J.; Ros, E.; Martínez-González, M.A.; Fitó, M.; Estruch, R.; Corella, D.; Fiol, M.; Gómez-Gracia, E.; Arós, F.; et al. Effect of a Mediterranean diet supplemented with nuts on metabolic syndrome status: One-year results of the PREDIMED randomized trial. Arch. Intern. Med. 2008, 168, 2449-2458.

58. Babio, N.; Bulló, M.; Basora, J.; Martínez-González, M.A.; Fernández-Ballart, J.; MárquezSandoval, F.; Molina, C.; Salas-Salvadó, J. Adherence to the Mediterranean diet and risk of metabolic syndrome and its components. Nutr. Metab. Cardiovasc. Dis. 2009, 19, 563-570. 
59. Martínez-González, M.A.; Fernández-Jarne, E.; Serrano-Martínez, M.; Wright, M.; Gomez-Gracia, E. Development of a short dietary intake questionnaire for the quantitative estimation of adherence to a cardioprotective Mediterranean diet. Eur. J. Clin. Nutr. 2004, 58, 1550-1552.

60. Rumawas, M.E.; Dwyer, J.Y.; Mckeown, N.M.; Meigs, J.B.; Rogers, G.; Jacques, P.F. The development of the Mediterranean-style dietary pattern score and its application to the American diet in the Framingham Offspring Cohort. J. Nutr. 2009, 139, 1150-1156.

61. Paletas, K.; Athanasiadou, E.; Sarigianni, M.; Paschos, P.; Kalogirou, A.; Hassapidou, M.; Tsapas, A. The protective role of the Mediterranean diet on the prevalence of metabolic syndrome in a population of Greek obese subjects. J. Am. Coll. Nutr. 2010, 29, 41-45.

62. Kesse-Guyot, E.; Ahluwalia, N.; Lassale, C.; Hercberg, S.; Fezeu, L.; Lairon, D. Adherence to Mediterranean diet reduces the risk of metabolic syndrome: A 6-year prospective study. Nutr. Metab. Cardiovasc. Dis. 2013, 23, 677-683.

63. Issa, C.; Darmon, N.; Salameh, P.; Maillot, M.; Batal, M.; Lairon, D. Mediterranean diet pattern with low consumption of liquid sweets and refined cereals is negatively associated with adiposity in adults from rural Lebanon. Int. J. Obes. (Lond.) 2011, 35, 251-258.

64. Schroder, H.; Marrugat, J.; Vila, J.; Covas, M.I.; Elosua, R. Adherence to the traditional Mediterranean diet is inversely associated with body mass index and obesity in a Spanish population. J. Nutr. 2004, 134, 3355-3361.

65. Trichopoulou, A.; Naska, A.; Orfanos, P.; Trichopoulos, D. Mediterranean diet in relation to body mass index and waist-to-hip ratio: The Greek European Prospective Investigation into Cancer and Nutrition Study. Am. J. Clin. Nutr. 2005, 82, 935-940.

66. Panagiotakos, D.B.; Chrysohoou, C.; Pitsavos, C.; Stefanadis, C. Association between the prevalence of obesity and adherence to the Mediterranean diet: The ATTICA study. Nutrition 2006, 22, 449-456.

67. Mendez, M.A.; Popkin, B.M.; Jakszyn, P.; Berenguer, A.; Tormo, M.J.; Sanchéz, M.J.; Quirós, J.R.; Pera, G.; Navarro, C.; Martinez, C.; et al. Adherence to a Mediterranean diet is associated with reduced 3-year incidence of obesity. J. Nutr. 2006, 136, 2934-2938.

68. Panagiotakos, D.B.; Polystipioti, A.; Papairakleous, N.; Polychronopoulos, E. Long-Term adoption of a Mediterranean diet is associated with a better health status in elderly people; a cross-sectional survey in Cyprus. Asia Pac. J. Clin. Nutr. 2007, 16, 331-337.

69. Beunza, J.J.; Toledo, E.; Hu, F.B.; Bes-Rastrollo, M.; Serrano-Martínez, M.; Sánchez-Villegas, A.; Martínez, J.A.; Martínez-González, M.A. Adherence to the Mediterranean diet, long-term weight change, and incident overweight or obesity: The Seguimiento Universidad de Navarra (SUN) cohort. Am. J. Clin. Nutr. 2010, 92, 1484-1493.

70. Romaguera, D.; Norat, T.; Vergnaud, A.C.; Mouw, T.; May, A.M.; Agudo, A.; Buckland, G.; Slimani, N.; Rinaldi, S.; Couto, E.; et al. Mediterranean dietary patterns and prospective weight change in participants of the EPIC-PANACEA project. Am. J. Clin. Nutr. 2010, 92, 912-921.

71. Buckland, G.; González, C.A.; Agudo, A.; Vilardell, M.; Berenguer, A.; Amiano, P.; Ardanaz, E.; Arriola, L.; Barricarte, A.; Basterretxea, M.; et al. Adherence to the Mediterranean diet and risk of coronary heart disease in the Spanish EPIC Cohort Study. Am. J. Epidemiol. 2009, 170, $1518-1529$. 
72. Serra-Majem, L.; Ribas, L.; Ngo, J.; Ortega, R.M.; García, A.; Pérez-Rodrigo, C.; Aranceta, J. Food, youth and the Mediterranean diet in Spain. Development of KIDMED, Mediterranean Diet Quality Index in children and adolescents. Public Health Nutr. 2004, 7, 931-935.

73. Martínez-González, M.A.; García-Arellano, A.; Toledo, E.; Salas-Salvadó, J.; Buil-Cosiales, P.; Corella, D.; Covas, M.; Schröder, H.; Arós, F.; Gómez-Gracia, E.; et al. A 14-item Mediterranean diet assessment tool and obesity indexes among high-risk subjects: The PREDIMED trial. PLoS One 2012, 7, e43134, doi:10.1371/journal.pone.0043134.

74. Panagiotakos, D.B.; Pitsavos, C.; Arvaniti, F.; Stefanadis, C. Adherence to the Mediterrranean food pattern predicts the prevalence of hypertension, hypercholesterolemia, diabetes and obesity, among healthy adults; the accuracy of the MedDietScore. Prev. Med. 2007, 44, 335-340.

75. Martínez-González, M.A.; García-López, M.; Bes-Rastrollo, M.; Toledo, E.; Martínez-Lapiscina, E.H.; Delgado-Rodriguez, M.; Vazquez, Z.; Benito, S.; Beunza, J.J. Mediterranean diet and the incidence of cardiovascular disease: A Spanish cohort. Nutr. Metab. Cardiovasc. Dis. 2011, 21, 237-244.

76. Menotti, A.; Alberti-Fidanza, A.; Fidanza, F. The association of the Mediterranean Adequacy Index with fatal coronary events in an Italian middle-aged male population followed for 40 years. Nutr. Metab. Cardiovasc. Dis. 2012, 22, 369-375.

77. Fidanza, F.; Alberti, A.; Lanti, M.; Menotti, A. Mediterranean adequacy index: Correlation with 25-year mortality from coronary heart disease in the Seven Countries Study. Nutr. Metab. Cardiovasc. Dis. 2004, 14, 254-258.

78. Guallar-Castillón, P.; Rodríguez-Artalejo, F.; Tormo, M.J.; Sánchez, M.J.; Rodríguez, L.; Quirós, J.R.; Navarro, C.; Molina, E.; Martínez, C.; Marín, P.; et al. Major dietary patterns and risk of coronary heart disease in middle-aged persons from a Mediterranean country: The EPIC-Spain cohort study. Nutr. Metab. Cardiovasc. Dis. 2012, 22, 192-199.

79. Grundy, S.M.; Cleeman, J.I.; Daniels, S.R.; Donato, K.A.; Eckel, R.H.; Franklin, B.A.; Gordon, D.J.; Krauss, R.M.; Savage, P.J.; Smith, S.C., Jr.; et al. Diagnosis and management of the metabolic syndrome: An american heart association/national heart, lung, and blood institute scientific statement. Circulation 2005, 112, 2735-2752.

80. Kastorini, C.M.; Milionis, H.J.; Esposito, K.; Giugliano, D.; Goudevenos, J.A.; Panagiotakos, D.B. The effect of Mediterranean diet on metabolic syndrome and its components. A metaanalysis of 50 studies and 534,906 individuals. J. Am. Coll. Cardiol. 2011, 57, 1299-1313.

81. Martinez-Gonzalez, M.A.; Bes-Rastrollo, M. Dietary patterns, Mediterranean diet, and cardiovascular disease. Curr. Opin. Lipidol. 2014, 25, 20-26.

82. Jenkins, D.J.; Kendall, C.W.; Marchie, A.; Faulkner, D.A.; Wong, J.M.; de Souza, R.; Emam, A.; Parker, T.L.; Vidgen, E.; Lapsley, K.G.; et al. Effects of a dietary portfolio of cholesterol-lowering foods vs. lovastatin on serum lipids and C-reactive protein. JAMA 2003, 290, 502-510.

83. American Public Health Association (APHA). Available online: http://action.apha.org/site/ PageServer?pagename=Obesity_Infographic (accessed on 25 January 2014).

84. Evert, A.B.; Boucher, J.L.; Cypress, M.; Dunbar, S.A.; Franz, M.J.; Mayer-Davis, E.J.; Neumiller, J.J.; Nwankwo, R.; Verdi, C.L.; Urbanski, P.; et al. Nutrition therapy Recommendations for the management of adults with diabetes. Diabetes Care 2013, 36, 3821-3842. 
85. Hu, F.; Willett, W.C. Optimal diets for prevention of coronary heart disease. JAMA 2002, 288, 2569-2578.

86. Grundy, S.M. Lipids, nutrition and coronary heart disease. In Atherosclerosis and Coronary Artery Disease; Fuster, V., Ross, R., Topol, E.J., Eds.; Lippincott-Raven: Philadelphia, PA, USA, 1996.

87. Mente, A.; de Koning, L.; Shannon, H.S.; Anand, S.S. A systematic review of the evidence supporting a causal link between dietary factors and coronary heart diseases. Arch. Intern. Med. 2009, 169, 659-669.

88. ESC/EAS. Guidelines for the management of dyslipidaemias. Eur. Heart J. 2011, 32, 1769-1818.

89. Connor, W.E.; Connor, S.L. Should a low-fat, high-carbohydrate diet be recommended for everyone? The case for a low-fat, high-carbohydrate diet. N. Engl. J. Med. 1997, 337, 562-563.

90. Jequier, E.; Bray, G.A. Low-Fat diets are preferred? Am. J. Med. 2002, 113, 41S-46S.

91. Ferro-Luzzi, A.; Branca, F. Mediterranean Diet, Italian-style: Prototype of a healthy diet. Am. J. Clin. Nutr. 1995, 61, 1338S-1345S.

92. Chowdhury, R.; Warnakula, S.; Kunutsor, S.; Crowe, F.; Ward, H.A.; Johnson, L.; Franco, O.H.; Butterworth, A.S.; Forouhi, N.G.; Thompson, S.G.; et al. Association of dietary, circulating, and supplement fatty acids with coronary risk: A Systematic review and meta-analysis. Ann. Intern. Med. 2014, 160, 398-406.

93. McManus, K.; Antinoro, L.; Sacks, F. A randomized controlled trial of a moderate-fat, low-energy diet compared with a low fat, low-energy diet for weight loss in overweight adults. Int. J. Obes. 2001, 25, 1503-1511.

94. Alberti-Fidanza, A.; Fidanza, F.; Chiuchiù, M.P.; Verducci, G.; Fruttini, D. Dietary studies on two rural Italian population groups of the Seven Countries Study. 3. Trend of food and nutrient intake from 1960 to 1991. Eur. J. Clin. Nutr. 1999, 53, 854-860.

95. Programa de actividades preventivas y promoción de la salud (PAPPS). Guía para ayudar a promover una alimentación saludable. In Grupo de Educación Sanitaria y Promoción de la Salud; SEMFYC: Barcelona, Spain, 2000.

96. OMS 2004 Organización Mundial de la Salud. Adherencia a los Tratamientos a Largo Plazo; Pruebas para la Acción. World Health Oranization: Geneva, Switerzland, 2004.

(C) 2014 by the authors; licensee MDPI, Basel, Switzerland. This article is an open access article distributed under the terms and conditions of the Creative Commons Attribution license (http://creativecommons.org/licenses/by/3.0/). 